\title{
ENERGETIC HYDROGEN AND OXYGEN ATOMS OBSERVED ON THE NIGHTSIDE OF MARS
}

\author{
A. GALLI ${ }^{1, *}$, P. WURZ ${ }^{1}$, S. BARABASH ${ }^{2}$, A. GRIGORIEV ${ }^{2}$, H. GUNELL ${ }^{2}$, \\ R. LUNDIN ${ }^{2}$, M. HOLMSTRÖM ${ }^{2}$ and A. FEDOROV ${ }^{3}$ \\ ${ }^{1}$ Physikalisches Institut, Sidlerstrasse 5, CH-3012, Bern, Switzerland \\ ${ }^{2}$ Swedish Institute of Space Physics, Box 812,SE-981 28 Kiruna, Sweden \\ ${ }^{3}$ Centre d'Etude Spatiale des Rayonnements, BP-4346, F-31028 Toulouse, France \\ (*Author for correspondence: E-mail: galli@space.unibe.ch)
}

(Received 23 June 2006; Accepted in final form 25 October 2006)

\begin{abstract}
We present measurements of energetic hydrogen and oxygen atoms (ENAs) on the nightside of Mars detected by the neutral particle detector (NPD) of ASPERA-3 on Mars Express. We focus on the observations for which the field-of-view of NPD was directed at the nightside of Mars or at the region around the limb, thus monitoring the flow of ENAs towards the nightside of the planet. We derive energy spectra and total fluxes, and have compiled maps of hydrogen ENA outflow. The hydrogen ENA intensities reach $10^{5} \mathrm{~cm}^{-2} \mathrm{sr}^{-1} \mathrm{~s}^{-1}$, but no oxygen ENA signals above the detection threshold of $10^{4} \mathrm{~cm}^{-2} \mathrm{sr}^{-1} \mathrm{~s}^{-1}$ are observed. These intensities are considerably lower than most theoretical predictions. We explain the discrepancy as due to an overestimation of the charge-exchange processes in the models for which too high an exospheric density was assumed. Recent UV limb emission measurements (Galli et al., this issue) point to a hydrogen exobase density of $10^{10} \mathrm{~m}^{-3}$ and a very hot hydrogen component, whereas the models were based on a hydrogen exobase density of $10^{12} \mathrm{~m}^{-3}$ and a temperature of $200 \mathrm{~K}$ predicted by Krasnopolsky and Gladstone (1996). Finally, we estimate the global atmospheric loss rate of hydrogen and oxygen due to the production of ENAs.
\end{abstract}

Keywords: Martian atmosphere, atmospheric loss, energetic neutral atoms

\section{Introduction}

The emission of energetic neutral atoms (ENAs) from Mars has been addressed by several models (Kallio et al., 1997; Holmström et al., 2002; Barabash et al., 2002; Lichtenegger et al., 2002; Gunell et al., 2006), but direct ENA measurements have become available only since the orbit insertion of ESA's Mars Express (MEX) spacecraft in December 2003. ENA images can give us a global picture of the interaction processes between the solar wind and the Mars atmosphere. If interpreted correctly, they allow us to deduce the physical properties of the exosphere and to quantify atmospheric loss processes. The production of ENAs in itself is not a major channel of the atmospheric escape for hydrogen or oxygen, but it scales with the ion escape rate (Barabash et al., 2002). The first publications of ENA data from the ASPERA-3 experiment on MEX were concerned with the hydrogen ENAs seen on the dayside of Mars that are most probably neutralized solar wind protons; Futaana

Space Science Reviews (2006) 126: 267-297

DOI: $10.1007 / \mathrm{s} 11214-006-9088-8$

(C) Springer 2007 
et al. (2006a) reported on the backscattered ENA albedo, and Futaana et al. (2006b) presented a study of the subsolar ENA jet, an intense and highly directional stream of hydrogen ENAs emitted from the subsolar region of Mars. The work we present here is dedicated to those ENA signals that were measured when Mars Express was on the nightside of Mars. Most observations were made for ENAs flowing away from the Sun towards the tail of the martian magnetosphere when the aperture plane of the neutral particle detector (NPD) was in the ecliptic plane, directed towards the sunward hemisphere. After the introduction we briefly characterize the NPD with which the data were obtained (Section 2) and we show the observation conditions (Section 3). Section 4 is dedicated to the hydrogen ENA signals: we present energy spectra of tailward flowing hydrogen ENAs, and we compare the global image of integral H-ENA intensities to model predictions. In Section 5 we show that no oxygen ENA signal above the detection limit has been found, and in Section 6 we deduce the global production rates of hydrogen and oxygen ENAs from our measurements. We conclude with Section 7 where we try to answer the following three questions: Can we distinguish planetary from solar wind hydrogen ENAs at the martian nightside? How does the global image of ENA fluxes compare to theoretical models? What is the loss rate of planetary hydrogen and oxygen due to the production of ENAs?

Because Mars lacks a significant intrinsic magnetic field, the solar wind directly interacts with the upper parts of the neutral atmosphere. The induced magnetosphere boundary (IMB) forces the solar wind plasma to flow around the denser part of the neutral atmosphere, but the upper atmosphere and ionosphere extend far beyond the IMB. The IMB is defined as the stopping boundary for the solar wind, the interior of the IMB is dominated by plasma of planetary origin. The location of the IMB is variable, for high solar wind pressure it is shifted to lower altitudes: on the dayside ASPERA-3 detected solar wind ions down to $300 \mathrm{~km}$ above the surface (Lundin et al., 2004). The region outside the IMB, the magnetosheath, is dominated by the shocked solar wind plasma. Still further away from the planet (0.5 Mars radii $\left(R_{\mathrm{M}}\right)$ above the subsolar point according to the model of Kallio et al. (1997)) the bow shock separates the magnetosheath from the undisturbed solar wind. In regions directly exposed to the solar wind, the planetary atoms ionized by solar UV radiation, by charge-exchange or electron-impact processes, are picked up and are accelerated away from the planet in the solar wind electric field. This results in a strong erosion of the martian atmosphere. To quantify this loss process one has to measure local ion fluxes at many places inside the IMB and in the magnetosheath (Lundin et al., 1989; Dubinin et al., 2006a,b) or one can measure the flux of ENAs escaping the martian atmosphere to obtain an image from which a global estimate of the neutral escape can be made. Through forward modeling it is also possible to put constraints on the escape through pick-up ions. 
An ENA is the product of a charge-exchange collision between an accelerated ion and an ambient neutral atom (Wurz, 2000). The ENA intensity $J_{\mathrm{ENA}}$ can be described by the line-of-sight (LOS) integral

$$
J_{\mathrm{ENA}}=\sigma \int_{\mathrm{LOS}} d s n_{\mathrm{H}}(r) J_{\mathrm{p}}(s)
$$

through the martian exosphere. Equation (1) holds for the simple case of a stream of hydrogen ions $J_{\mathrm{p}}(s)$ that are neutralized in a pure hydrogen exosphere with density $n_{\mathrm{H}}(r)$. In general, the charge-exchange cross section $\sigma$ varies with ion energy and the ions may charge-exchange with other neutral species as well. Interpreting the data is therefore a complicated task as it requires models of the various ion populations. The exospheric density profiles of neutrals must also be known to interpret ENA data. A further complication is that MEX is not equipped with a magnetometer. In all studies of plasma data from the ASPERA-3 experiment the direction of the magnetic fields has to be inferred from Mars Global Surveyor (MGS) measurements or from indirect methods (Yamauchi et al., this issue).

For the NPD measurements we present in this study, 'energetic neutral atom' refers to velocities between 100 and $1000 \mathrm{~km} \mathrm{~s}^{-1}$, i.e., 0.1 to $10 \mathrm{keV}$ (see Section 2). We only consider hydrogen and oxygen ENAs; they are the most abundant ENA species (data from the ion mass analyzer of ASPERA-3 show that $\mathrm{O}^{+}$ions are more abundant than $\mathrm{O}_{2}^{+}$and $\mathrm{CO}_{2}^{+}$in the martian exosphere (Carlsson et al., 2006)), and other species, such as $\mathrm{CO}, \mathrm{CO}_{2}$, and $\mathrm{O}_{2}$, result in ENAs out of the NPD measurement range. He-ENAs are in principle detectable by NPD, and the $\mathrm{He}^{+}$ ion flux has been found to reach several $10^{6} \mathrm{~cm}^{-2} \mathrm{~s}^{-1}$ at some locations in the martian exosphere (Barabash et al., 1995). However, these ion fluxes do not result in He-ENA intensities above the NPD detection threshold of $10^{4} \mathrm{~cm}^{-2} \mathrm{sr}^{-1} \mathrm{~s}^{-1}$ because the cross section for the charge-exchange between a $1 \mathrm{keV} \mathrm{He}^{+}$and a neutral hydrogen atom is two orders of magnitude lower (Macias et al., 1983) than the cross section for the reaction between a proton and a neutral hydrogen atom (see Figure 1). In the NPD energy range the neutral gas species to be taken into account for ENA generation are $\mathrm{H}, \mathrm{H}_{2}$, and $\mathrm{O}$. The neutral hydrogen population is the most important species because its density in the exosphere is higher than those of any other species due to its large scale height.

Energetic neutral hydrogen atoms observed at Mars either are solar wind protons that have been neutralized in the atmosphere (Futaana et al., 2006a, b) or they are planetary hydrogen atoms that have been ionized and accelerated in the ambient electro-magnetic fields before charge-exchanging with the neutral gas again. The most important reactions that produce a hydrogen ENA are (Lichtenegger et al., 2002):

$$
\begin{aligned}
& \mathrm{H}_{s w}^{+}+\mathrm{O} \rightarrow \mathrm{H}_{s w}^{\mathrm{ENA}}+\mathrm{O}^{+} \\
& \mathrm{H}_{s w}^{+}+\mathrm{H} \rightarrow \mathrm{H}_{s w}^{\mathrm{ENA}}+\mathrm{H}^{+} \\
& \mathrm{H}_{s w}^{+}+\mathrm{H}_{2} \rightarrow \mathrm{H}_{s w}^{\mathrm{ENA}}+\mathrm{H}_{2}^{+}
\end{aligned}
$$



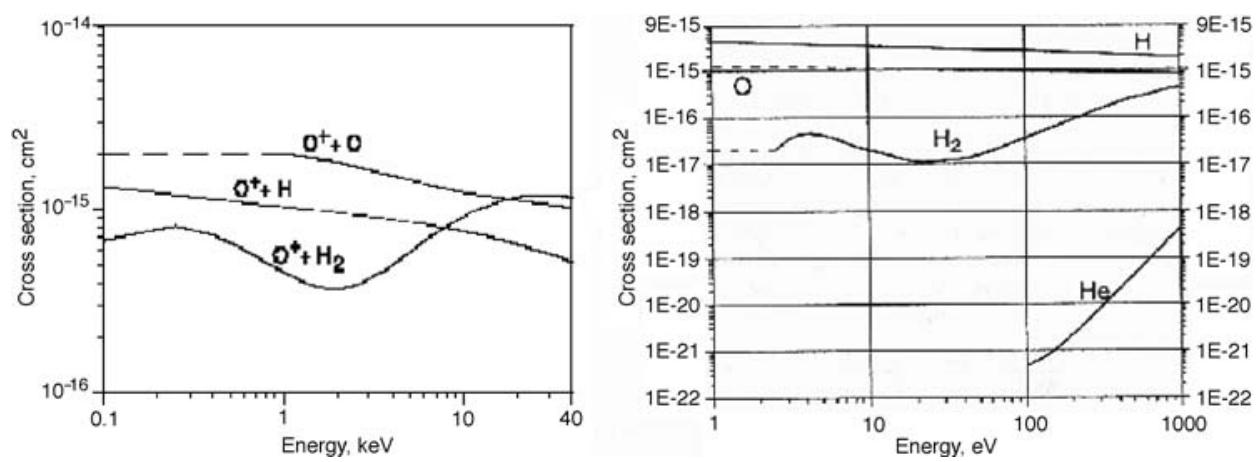

Figure 1. Cross sections of the most important charge-exchange reactions that produce oxygen (left panel) and hydrogen ENAs (right panel) in the martian exosphere. The diagrams are taken from Barabash et al. (2002) (left panel) and from Kallio et al. (1997) (right panel).

$$
\begin{aligned}
\mathrm{H}_{p l}^{+}+\mathrm{O} & \rightarrow \mathrm{H}_{p l}^{\mathrm{ENA}}+\mathrm{O}^{+} \\
\mathrm{H}_{p l}^{+}+\mathrm{H} & \rightarrow \mathrm{H}_{p l}^{\mathrm{ENA}}+\mathrm{H}^{+} \\
\mathrm{H}_{p l}^{+}+\mathrm{H}_{2} & \rightarrow \mathrm{H}_{p l}^{\mathrm{ENA}}+\mathrm{H}_{2}^{+}
\end{aligned}
$$

where $\mathrm{H}_{s w}$ stands for solar wind hydrogen and $\mathrm{H}_{p l}$ for planetary hydrogen. The right panel in Figure 1 shows the cross sections of the four charge-exchange reactions:

$$
\begin{aligned}
\mathrm{H}^{+}+\mathrm{O} \rightarrow \mathrm{H}^{\mathrm{ENA}}+\mathrm{O}^{+} \\
\mathrm{H}^{+}+\mathrm{H} \rightarrow \mathrm{H}^{\mathrm{ENA}}+\mathrm{H}^{+} \\
\mathrm{H}^{+}+\mathrm{H}_{2} \rightarrow \mathrm{H}^{\mathrm{ENA}}+\mathrm{H}_{2}^{+} \\
\stackrel{\text { and }}{\mathrm{H}^{+}+\mathrm{He} \rightarrow \mathrm{H}^{\mathrm{ENA}}+\mathrm{He}^{+}}
\end{aligned}
$$

The neutral helium will not be taken into account in the subsequent discussions because of its low charge-exchange cross section. The left panel in Figure 1 shows the charge-exchange cross sections for the reactions

$$
\begin{aligned}
& \mathrm{O}^{+}+\mathrm{O} \rightarrow \mathrm{O}^{\mathrm{ENA}}+\mathrm{O}^{+} \\
& \mathrm{O}^{+}+\mathrm{H} \rightarrow \mathrm{O}^{\mathrm{ENA}}+\mathrm{H}^{+} \\
& \mathrm{O}^{+}+\mathrm{H}_{2} \rightarrow \mathrm{O}^{\mathrm{ENA}}+\mathrm{H}_{2}^{+}
\end{aligned}
$$

In all cases the oxygen ENAs originate from Mars.

In analyzing the measurements of nightside ENAs we try to find answers to the three following questions:

1. Can we distinguish planetary from solar wind hydrogen ENAs?

If we want to quantify the erosion processes in the martian atmosphere we need to image the planetary hydrogen ENAs. On the dayside, however, the measured H-ENA signals are dominated by neutralized solar wind protons (Futaana et al., 2006a, b). Lichtenegger et al. (2002) suggest that on the nightside planetary 
hydrogen ENAs constitute up to $15 \%$ of the tailward flow of H-ENAs. Inside the IMB planetary H-ENAs should be separable from solar wind H-ENAs because of their lower energies, whereas planetary ENAs originating in the outer magnetosheath and upstream of the bow shock are expected to have higher energies than that of solar wind ENAs.

2. Are the global ENA images consistent with theoretical predictions or do we need to revise the model input parameters?

The temperature and density of the neutral atomic hydrogen in the exosphere, the strength of the solar wind, and the location of the bow shock and of the induced magnetosphere boundary (see Figure 2), are the most important parameters (Holmström et al., 2002) for the empirical model of Kallio et al. (1997) of the solar wind-Mars interaction. This model in general provides a similar global flux distribution as the only parameterized model available (Gunell et al., 2006).

3. What is the global production rate of planetary hydrogen and oxygen ENAs? How does it compare to measured ion escape rates (Lundin et al., 1989) and to models of atmospheric escape processes (Kim et al., 1998; Lammer et al., 2005)? The tailward flux of planetary ENAs, integrated over the planet, gives a minimum estimate of the present loss rate of the martian atmosphere because the speed of the detected ENAs by far exceeds the escape velocity. Moreover, since the global production rate of ENAs depends on the available flux of ions, it can be used as a proxy for the global escape rate of ions as proposed by Barabash et al. (2002).

\section{Instrumentation and Data Analysis}

The ASPERA-3 instrument on board the MEX spacecraft comprises four different sensors. The ion mass analyzer (IMA) and the electron spectrometer (ELS) are used to measure local ion and electron densities, the NPD and the neutral particle imager (NPI) are used to detect energetic neutral hydrogen and oxygen atoms (for a more detailed description of all four sensors see for instance Barabash and Lundin (2006) or Barabash et al. (2004)). In the current report we restrict ourselves to ENA data that were measured by NPD. The NPD consists of two identical sensors NPD1 and NPD2 that are sensitive to ENAs in the energy range of 0.1 to $10 \mathrm{keV}$. The velocity of an incident particle can be reconstructed from the time-of-flight (TOF) between start and stop surface; each coincidence with one start and one stop pulse within the TOF range of $2048 \mathrm{~ns}$ is sampled in the corresponding TOF bin of 8 ns width. Each NPD sensor has one start and three stop surfaces, which provide an angular resolution of roughly $30^{\circ}$ in azimuthal direction and $4^{\circ}$ in elevation direction. Together, these six azimuth channels give an instantaneous field of view of NPD of $180^{\circ} \times 4^{\circ}$. The upper panel in Figure 3 shows a spacecraft-centered view from Mars orbit of the six NPD channels, numbered from NPD1_0 to NPD2_2.

Integration times of typically ten minutes are required to obtain a reliable TOF spectrum of ENAs in units of counts $\mathrm{s}^{-1}$. First, we estimate the background of accidentals caused by UV photons and subtract it from the measured TOF spectrum. 

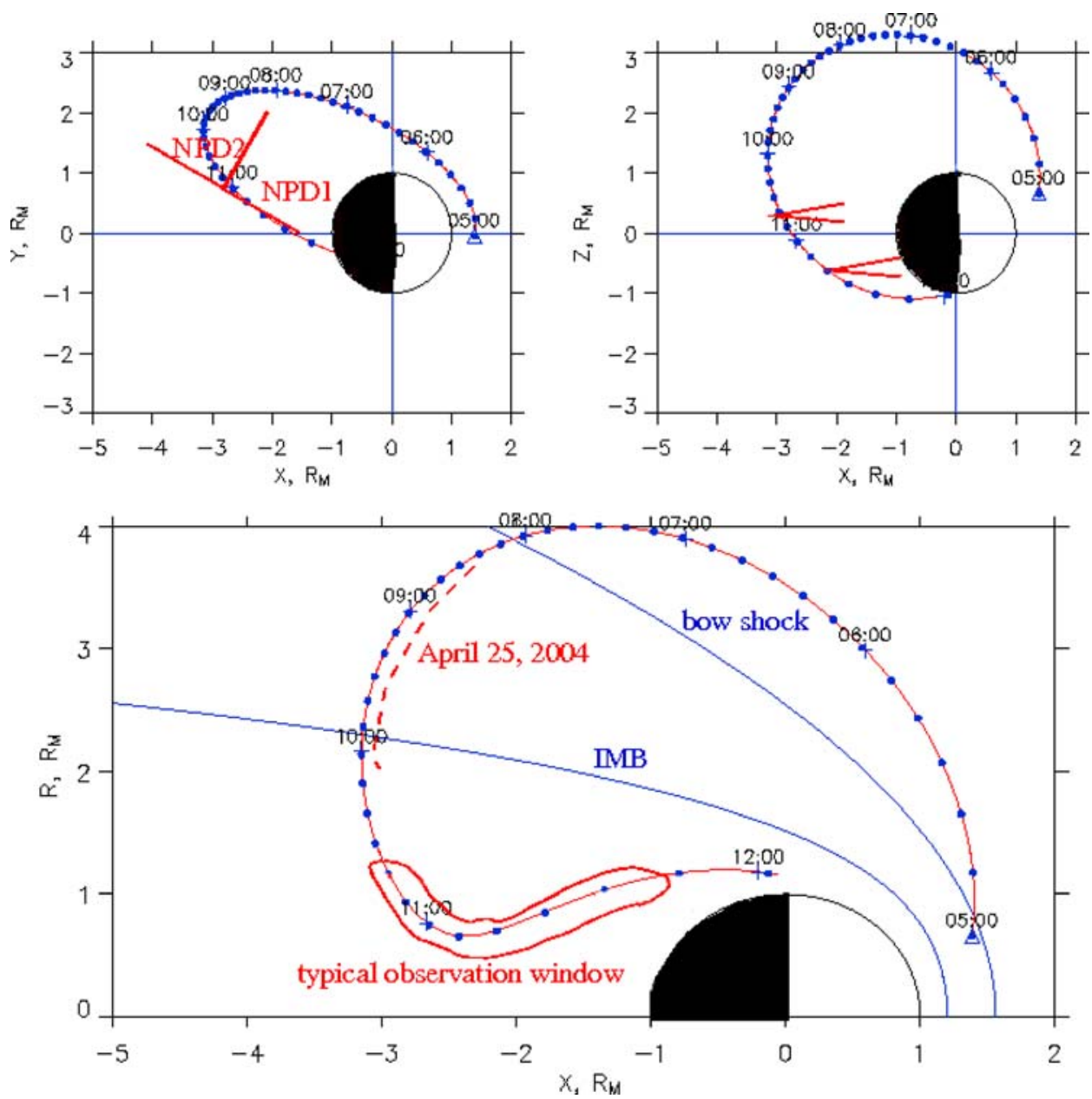

Figure 2. Typical NPD observation configuration. The time tags apply only to the orbit of April 9, 2004 (blue dots), but the shape of the orbit is representative for all orbits in April and May 2004. The upper panels show projections of the typical orbit onto the XY and the XZ plane, whereas in the lower panel the orbit is plotted in cylindrical coordinates, $R=\sqrt{Y^{2}+Z^{2}}$ being the distance to the MarsSun-line $X$. The red wedges in the upper panels denote the fields-of-view of the two NPD sensors. In the lower panel the inner blue curve denotes the IMB, the outer curve denotes the bow shock between the undisturbed solar wind and the magnetosheath (Kallio et al., 1997). Red encircled is the typical observation time of about half an hour (see Figure 3, upper panel, for a spacecraft-centered view).

The accidental count rates are spread equally across each TOF bin as they result from two uncorrelated UV photons that trigger a start and a stop pulse (see the dotted line in Figure 3). The height of the background is identified at the TOF bins from 200 to 256 that correspond to energies below $0.1 \mathrm{keV}$ for which NPD is insensitive to ENAs. If an ENA signal beyond the background is recognizable, we apply a low-pass filter to eliminate any signal with periods shorter than $10 \mathrm{TOF}$ 

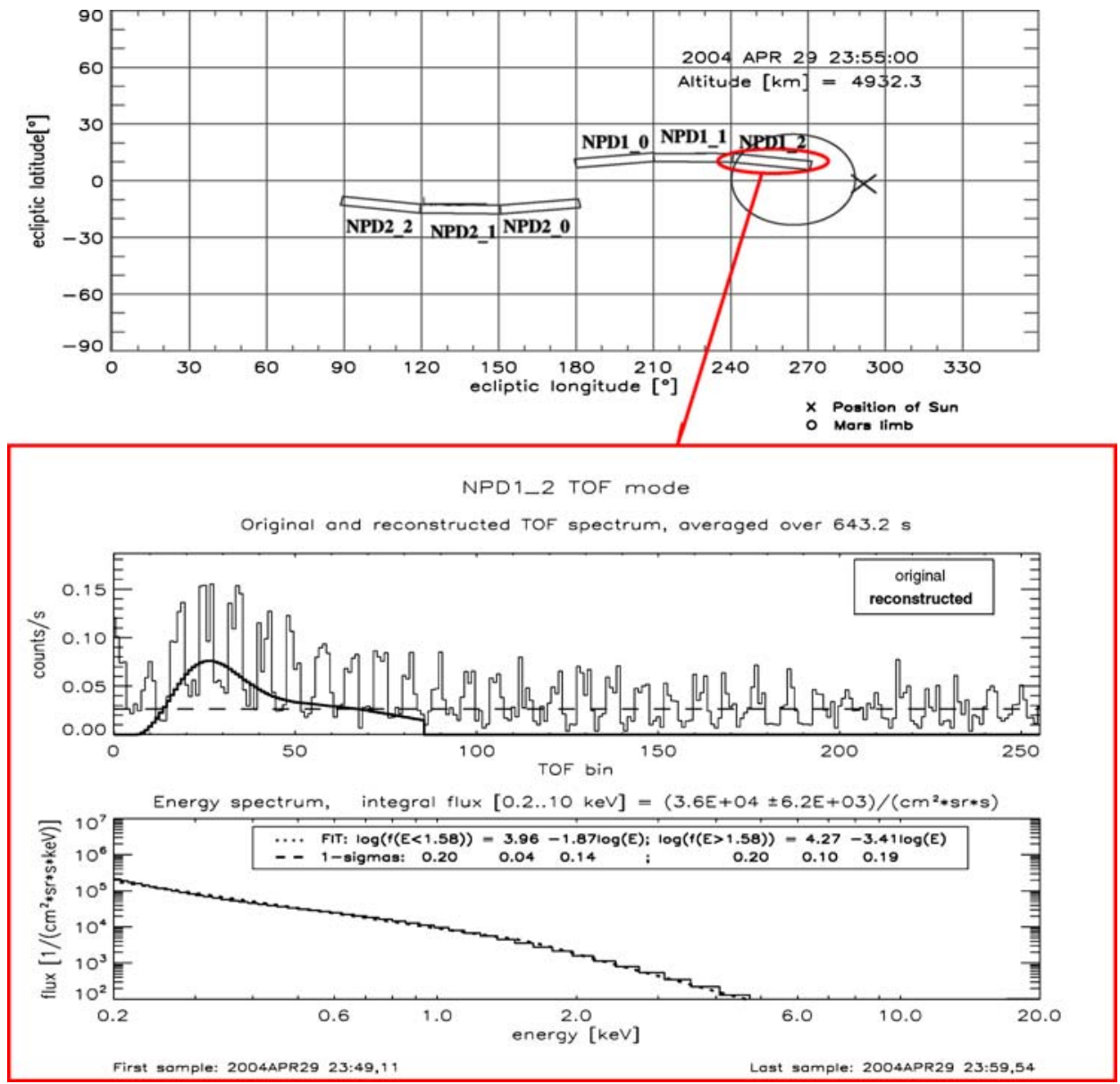

Figure 3. Hydrogen ENAs measured by ASPERA-3/NPD during a 10 min observation period on April 29, 2004. The upper panel gives the observation direction in the ecliptic reference frame, the spacecraft position is the same as in Figure 2 (red encircled area). The middle panel shows the measured (thin line) and the reconstructed (bold line) TOF spectrum. The peak between TOF bins 20 and 100 is due to hydrogen ENAs, the flat noise level of 0.03 counts per second is due to coincident UV photons (dashed line). The lower panel shows the reconstructed differential intensity that corresponds to the reconstructed TOF-signal (see Section 2).

bins, including the harmonic noise caused by the sensor electronics (see, e.g., the measured TOF spectrum in Figure 3). We then invert the instrument response by searching for an optimal fit function (the reconstructed TOF spectrum shown as bold curve in the middle panel of Figure 3), which, applied to the instrument response function, comes closest to the measured TOF spectrum. Finally, the reconstructed TOF spectrum is converted to a differential intensity energy spectrum in units of $\mathrm{cm}^{-2} \mathrm{sr}^{-1} \mathrm{~s}^{-1} \mathrm{keV}^{-1}$ (lower panel of Figure 3) by dividing the count rate of each bin by the product of geometrical factor, energy-dependent efficiency and bin width 
in $\mathrm{keV}^{-1}$, assuming either hydrogen or oxygen particles (see Galli et al., 2006 for more details). Throughout this work, integral ENA intensities in units of $\mathrm{cm}^{-2}$ $\mathrm{sr}^{-1} \mathrm{~s}^{-1}$ are to be understood as differential intensity energy spectra integrated from 0.2 to $10 \mathrm{keV}$.

\section{Observation Geometry}

For the present analysis we have included all available NPD measurements from 2004 for which the NPD sensor was measuring ENAs at the nightside of Mars with a sufficiently high TOF resolution. These requirements limit our data base to the time period from February 10, 2004, to May 9, 2004. Within this period we have 21 different observation occasions, amounting to a total of ten hours of observation time. Figure 2 shows the observation configuration that is typical for the entire set of data, with the exception of the three measurements in February. The martian nightside is the black hemisphere, the Sun direction is in all images the positive $x$-axis. The blue dotted line indicates the spacecraft orbit, the red wedges (upper panels) indicate the NPD field-of-view directions projected onto the XY and the XZ plane. Usually, NPD1 was directed at the martian nightside while NPD2 was directed away from Mars towards the tail of the magnetosheath (see also Figure 3 for a spacecraft-centered view). For Figure 2 we use the Mars Sun Orbit reference frame, for which the $+\mathrm{X}$ axis is the direction from Mars to the Sun and the $+\mathrm{Z}$ axis is perpendicular to the orbit plane of Mars. The orbit parameters are similar for most observation occasions, thus, they can easily be compared to each other. Unfortunately, NPD was switched on only after entering the region inside the IMB, with the one exception of April 25 (red dashed curve in Figure 2). This is the only example where we see the tailward flow of ENAs in the region between the bow shock and the IMB. On all occasions the instrument had to be switched off before crossing the terminator to protect the NPD from direct sunlight.

\section{Hydrogen ENAs}

Hydrogen ENAs have been detected in the majority of NPD measurements on the nightside of Mars. With the exception of the three dates in February 2004 and the one measurement on April 25, 2004, all measurements were made inside the IMB (see Figure 2) at altitudes of at most $2 R_{\mathrm{M}}$ above Mars. In the following presentation of results, we first define the typical ENA differential intensity energy spectrum measured inside the IMB and at the IMB itself (Section 4.1), and we compare this spectrum to the neutralized solar wind protons measured on the dayside of Mars (Section 4.2). Then we present the only spectrum measured in the magnetosheath, which clearly differs from the typical IMB spectrum (Section 4.3). 
Finally, we construct a global picture of integral H-ENA intensities and interpret our measurements by comparing these values to theoretical predictions (Section 4.4).

\subsection{The Typical Spectrum of Hydrogen ENAs}

Figure 3 shows the hydrogen ENA spectrum detected in channel NPD1_2 on April 29, 2004, when the spacecraft was inside the IMB, $5000 \mathrm{~km}$ above the martian nightside surface. As usual, NPD1 was directed towards the sunward hemisphere, whereas NPD2 was pointed away from Mars towards the tail of the magnetosheath. As we shall see (Equation (3)), the shape of this ENA spectrum with a weak roll-over at $1.5 \mathrm{keV}$ is typical for all tailward flowing ENAs within the IMB. The integral intensity of $(3.6 \pm 0.6) \times 10^{4} \mathrm{~cm}^{-2} \mathrm{sr}^{-1} \mathrm{~s}^{-1}$ is rather low compared to other ENA signals because the field-of-view is directed at the planetary disk itself. In Section 4.4 we show that the intensities of tailward flowing H-ENAs vary between the detection limit and several $10^{5} \mathrm{~cm}^{-2} \mathrm{sr}^{-1} \mathrm{~s}^{-1}$, the highest intensities are detected around the Mars limb towards the Sun.

As expected, the ENA streams from the nightside towards the sunward hemisphere, detected with NPD2, are even weaker, bordering to the detection threshold of $10^{4} \mathrm{~cm}^{-2} \mathrm{sr}^{-1} \mathrm{~s}^{-1}$. The roll-over of the energy spectrum lies at lower energies between 0.5 and $1 \mathrm{keV}$, but there are less than 10 useful spectra to define a typical spectrum of sunward flowing ENAs.

The energy spectrum of the tailward ENA signals, on the other hand, can be well described by a two-component power law with two different slopes $a_{1}, b_{1}$, and a roll-over $c$ :

$$
j(E)= \begin{cases}a_{0} E^{a_{1}} & \text { for } E<c \\ b_{0} E^{b_{1}} & \text { for } E \geq c\end{cases}
$$

The two-component power law in Equation (2) has been chosen because it reproduces the measurements well; it is inappropriate for only 6 of the 59 wellconstrained spectra measured inside the IMB. There is no particular physical background to it, contrary to the Maxwell-Boltzmann parameterization shown in Figures 7 and 8 . If one averages over the remaining 53 energy spectra of tailward flowing hydrogen ENAs, excluding only the measurements in February 2004 and on April 25 (see Section 4.3) that were obtained when MEX was at the surface of the IMB or in the magnetosheath, one finds the following median values:

$$
j(E)= \begin{cases}a_{0} E^{-1.7} & \text { for } E<1.2 \mathrm{keV} \\ b_{0} E^{-2.7} & \text { for } E \geq 1.2 \mathrm{keV}\end{cases}
$$

To obtain these values for the typical spectrum in Equation (3) we averaged over all spectra of tailward flowing H-ENAs, whether they were observed from the planet itself or from the surrounding space. This is because we cannot define a typical eclipse or Mars limb spectrum. Most signals coming from the planet itself are 
April 30, 2004

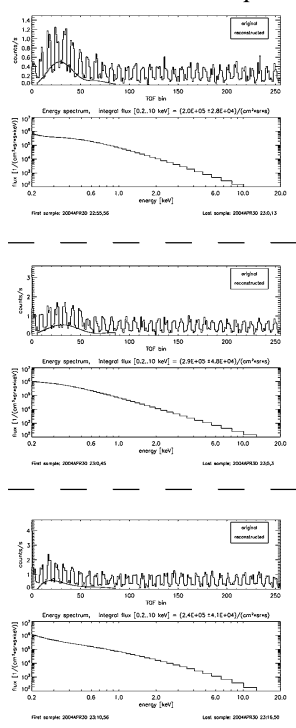

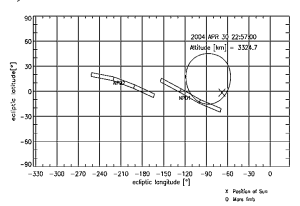

22:57 UT

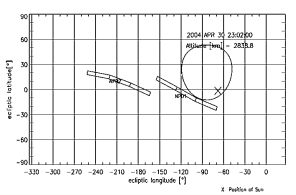

23:02 UT

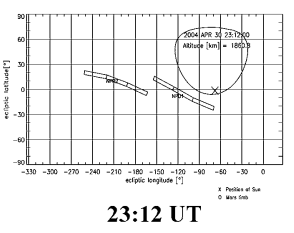

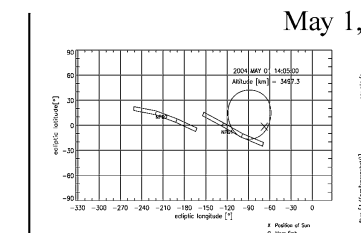

14:05 UT

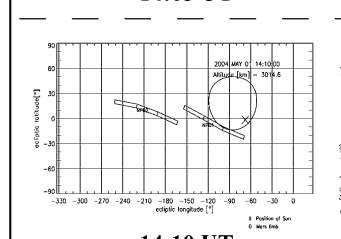

14:10 UT

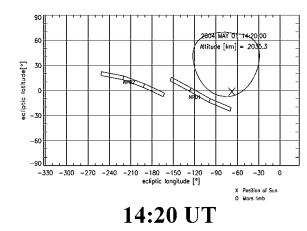

May 1,2004
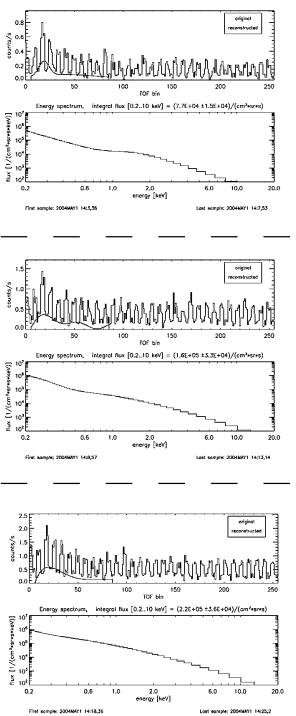

Figure 4. Temporal evolution of the H-ENA spectrum on the nightside of Mars for two consecutive measurements. The outer columns show the TOF and energy spectra measured by the NPD channel $1 \_2$, the inner columns show the corresponding observation configuration. The format of the spectra and of the position plots is identical to Figure 3, the spacecraft position is the same as in Figure 2 (red encircled area). The ENA spectra in the two upper rows for observations close to the Mars limb show a high variability between April 30 and May 1 . Once the field-of-view is out of the nightside and of the limb the spectra (bottom row) are similar to the typical nightside ENA spectrum as defined in Equation (3).

too weak (see the contour plots of integral intensities in Figure 9) to allow for a well-constrained energy spectrum, and the few well defined spectra show a high variability. This is illustrated by the time series in Figure 4 for two observations on April 30 and May 1, 2004 with almost identical orbit and viewing directions.

The energy spectra thus do not allow us to distinguish between two different ENA components inside the IMB. The only statistically significant pattern of the energy spectra inside the IMB is the correlation of the roll-over energy with distance to the Mars limb. Measurements made with the field-of-view covering the limb show a roll-over at higher energies than the signals observed from the planet itself or those far away from the limb. This is illustrated in Figure 5.

This trend reflects the variation in energy of the protons that give rise to the observed ENAs. Dubinin et al. (2006a, b) find, based on ion data obtained with IMA (see Section 2) in 2004, that inside the IMB the energy of $\mathrm{H}^{+}, \mathrm{H}_{2}^{+}, \mathrm{O}^{+}$, and $\mathrm{O}_{2}^{+}$ ions increases linearly with altitude from the planet because of the ambient electric field that accelerates the ions away from the planet. This increase in energy then stops in the magnetosheath (see Figure 9 in Dubinin et al. (2006b)). Outside the 


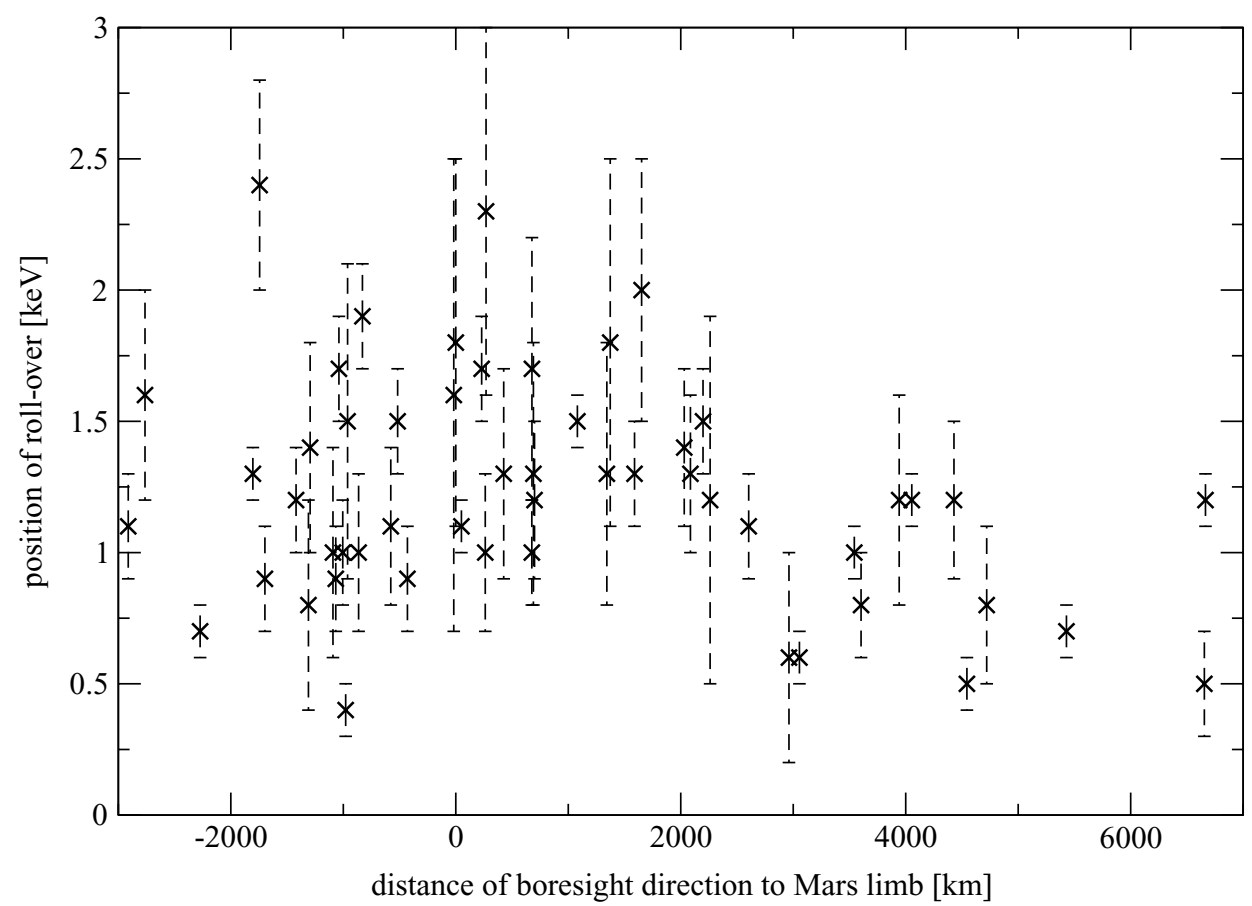

Figure 5. Place of the roll-over of observed H-ENA energy spectra plotted against the distance of the NPD LOS to the Mars limb. The roll-over is shifted to somewhat higher energies when the fieldof-view is close to the Mars limb. All these measurements were obtained when MEX was inside the IMB above the martian nightside (red encircled area in Figure 2).

bow shock only solar wind protons with roughly $1 \mathrm{keV}$ are available as parent ions for the production of ENAs. Based on these ion measurements one would predict the roll-over of ENA spectra in Figure 5 to be shifted to higher energies as the NPD LOS intersects ion populations at greater distances from the Mars exobase. Keep in mind, however, that unlike the local ion measurements the NPD measurements are to be interpreted as LOS integrals over regions of different ion populations. From Figure 5 it seems that the H-ENA signals observed from directions further than $2000 \mathrm{~km}$ away from the Mars limb are dominated by protons with solar wind energies that charge-exchange already on the dayside.

We conclude that the hydrogen ENA spectra measured inside the IMB are consistent with the ion measurements reported by Dubinin et al. (2006a, b). Unfortunately, they are no help to decide to what extent the observed H-ENA signals are due to planetary protons because the increase in energy with planetary distance is observed for planetary pick-up protons as well as for solar wind protons.

Contrary to the measurements in April and May 2004, the three observations in February 2004 were made when the spacecraft was at the boundary to the 
magnetosheath (see upper right panel in Figure 6). Again, it is not a priori clear whether the measured H-ENAs are neutralized solar wind protons or planetary ENAs flowing tailward along the IMB. The measured integral intensities are the highest in the entire data base as the NPD LOS is tangential to the IMB at some point close to the planet. The intensity reaches on all three occasions several $10^{5} \mathrm{~cm}^{-2}$ $\mathrm{sr}^{-1} \mathrm{~s}^{-1}$. The spectrum of the most intense ENA signal is shown in Figure 6. For this observation configuration the ENA signal in the NPD1 channels was probably more intense as they were directed to the Sun along the proton streamlines. Unfortunately, the NPD1 data of this observation are contaminated by Sun light. The median values of the 8 useful energy spectra cannot be discerned from the typical spectrum inside the IMB (Equation (3)). We find for the spectra at the boundary to the magnetosheath:

$$
j(E)= \begin{cases}a_{0} E^{-1.8} & \text { for } E<1.1 \mathrm{keV} \\ b_{0} E^{-2.9} & \text { for } E \geq 1.1 \mathrm{keV}\end{cases}
$$

\subsection{COMparison With SOlar Wind ENAs}

In order to compare to the typical night side ENA signal, Figure 7 shows an example of neutralized solar wind protons measured on the dayside of Mars on March 22, 2004. If an ENA spectrum reflects undisturbed solar wind protons that have been neutralized in the Mars exosphere before reaching the bow shock, we expect the ENAs to follow a maxwellian distribution as well. This also holds true for shocked solar wind protons in the magnetosheath as long as the protons are maxwellian with constant values for the thermal spread $k T$ and for the bulk flow velocity $\mathbf{v}_{s w}$. Contrary to $j(E)$ (Equation (3)) for the typical spectrum of ENAs on the nightside, the neutralized solar wind spectrum in Figure 7 therefore can be compared to a fit function that has a theoretical motivation. The resulting ENA energy spectrum $j(v)$ (with $\mathbf{v}_{\mathrm{ENA}}=\mathbf{v} \approx(v, 0,0)$ ) follows (Holmström et al., 2002)

$$
j(v)=\int_{\mathrm{LOS}} d s n_{\mathrm{H}}(r) \frac{v}{m} n_{p} \sigma v_{s w, x}\left(\frac{m}{2 \pi k T}\right)^{3 / 2} \exp \left(-\frac{m\left(\mathbf{v}-\mathbf{v}_{s w}\right)^{2}}{2 k T}\right),
$$

where $\sigma$ denotes the charge-exchange cross section, chosen to $2 \times 10^{-15} \mathrm{~cm}^{2}$ for the entire energy range of NPD (see Figure 1). $V_{s w, x}$ is the bulk velocity of the solar wind projected to the LOS of the detector, and $\Sigma_{\mathrm{H}}=\int d s n_{\mathrm{H}}(r)$ is the column density of neutral hydrogen along the LOS. Note that we have neglected the charge-exchange reactions with the neutral $\mathrm{O}$ and $\mathrm{H}_{2}$, which is only legitimate if the LOS does not intersect the atmosphere below the exobase. Moreover, Equation (5) is strictly correct only for an infinitesimally small spatial aperture angle of the instrument as we have approximated $\mathbf{v}_{\mathrm{ENA}} \approx(v, 0,0)$ for the velocity distribution of ENAs. $T(s), \mathbf{v}_{s w}(s)$, and $\mathrm{n}_{p}(s)$ vary over the LOS integral for observations deep inside the bow shock, (for a model of these spatial variations see e.g. Kallio et al., 

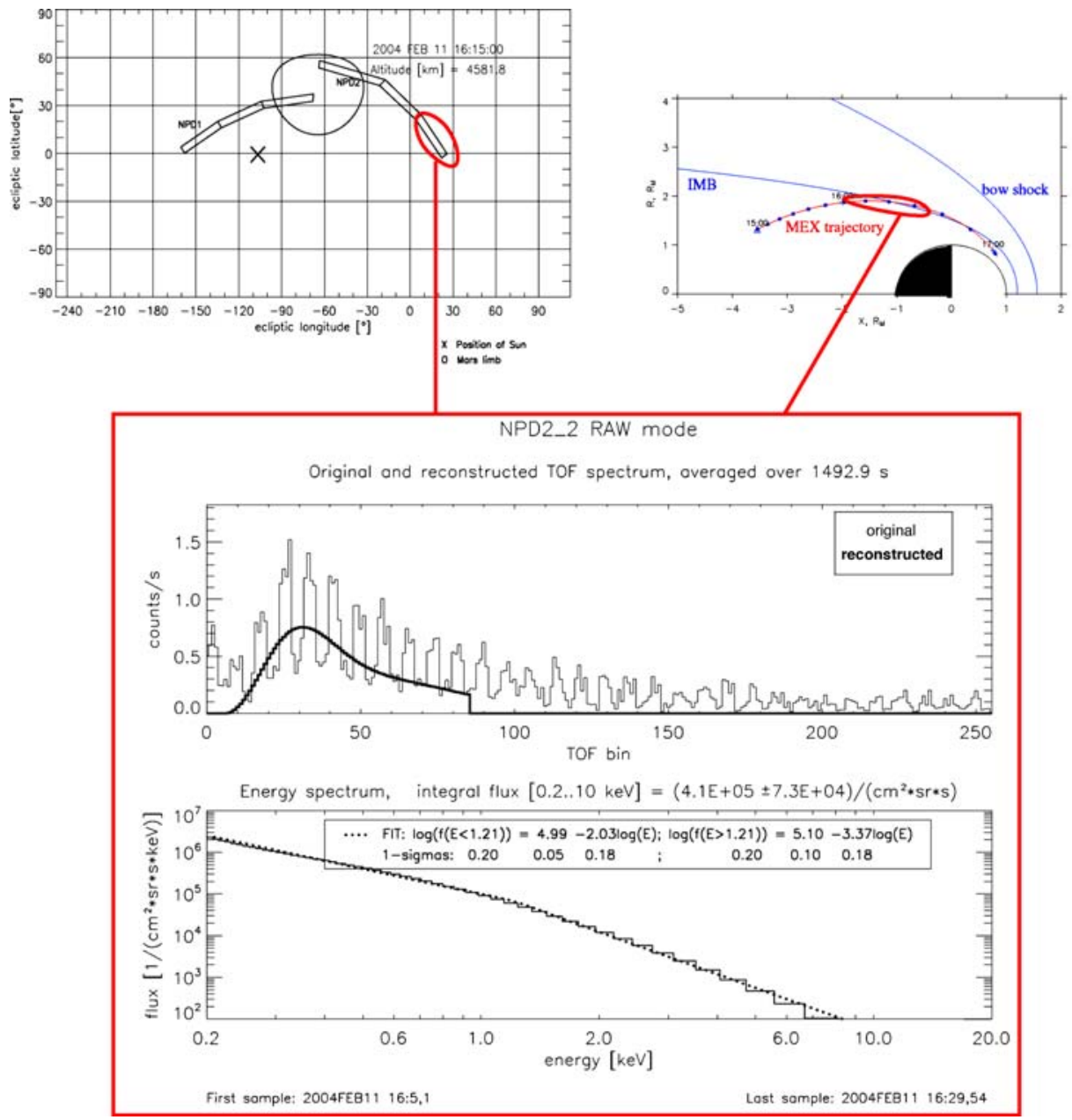

Figure 6. Hydrogen ENAs on the nightside of Mars, close to the tangential direction to the IMB surface. The data format is the same as in Figures 3 and 7. The energy spectrum (panel at the bottom) is very similar to the tailward flow of H-ENAs measured deep inside the IMB (see Figure 3).

1997), but in the case of solar wind protons with constant $T(s), \mathbf{v}_{s w}(s)$, and $\mathrm{n}_{p}(s)$ Equation (5) can be written in units of $\mathrm{cm}^{-2} \mathrm{sr}^{-1} \mathrm{~s}^{-1} \mathrm{keV}^{-1}$ as

$$
j(E)=c_{0} \sqrt{E} \exp \left(-c_{1} E+c_{2} \sqrt{E}\right) .
$$

Here, $c_{0}, c_{1}, c_{2}$ are three constants that depend on the thermal spread, the bulk flow velocity, the density of the solar wind, and on the hydrogen column density $\Sigma_{\mathrm{H}}$ along the LOS.

If we optimize the parameters $c_{0}, c_{1}, c_{2}$ in Equation (6) for the ENA spectrum of March 22, 2004, we obtain the fit that is plotted as dashed line in Figure 7. It implies a thermal spread of $k T=93 \mathrm{eV}$ and a bulk velocity of $v_{s w, x}=420 \mathrm{~km}$ 


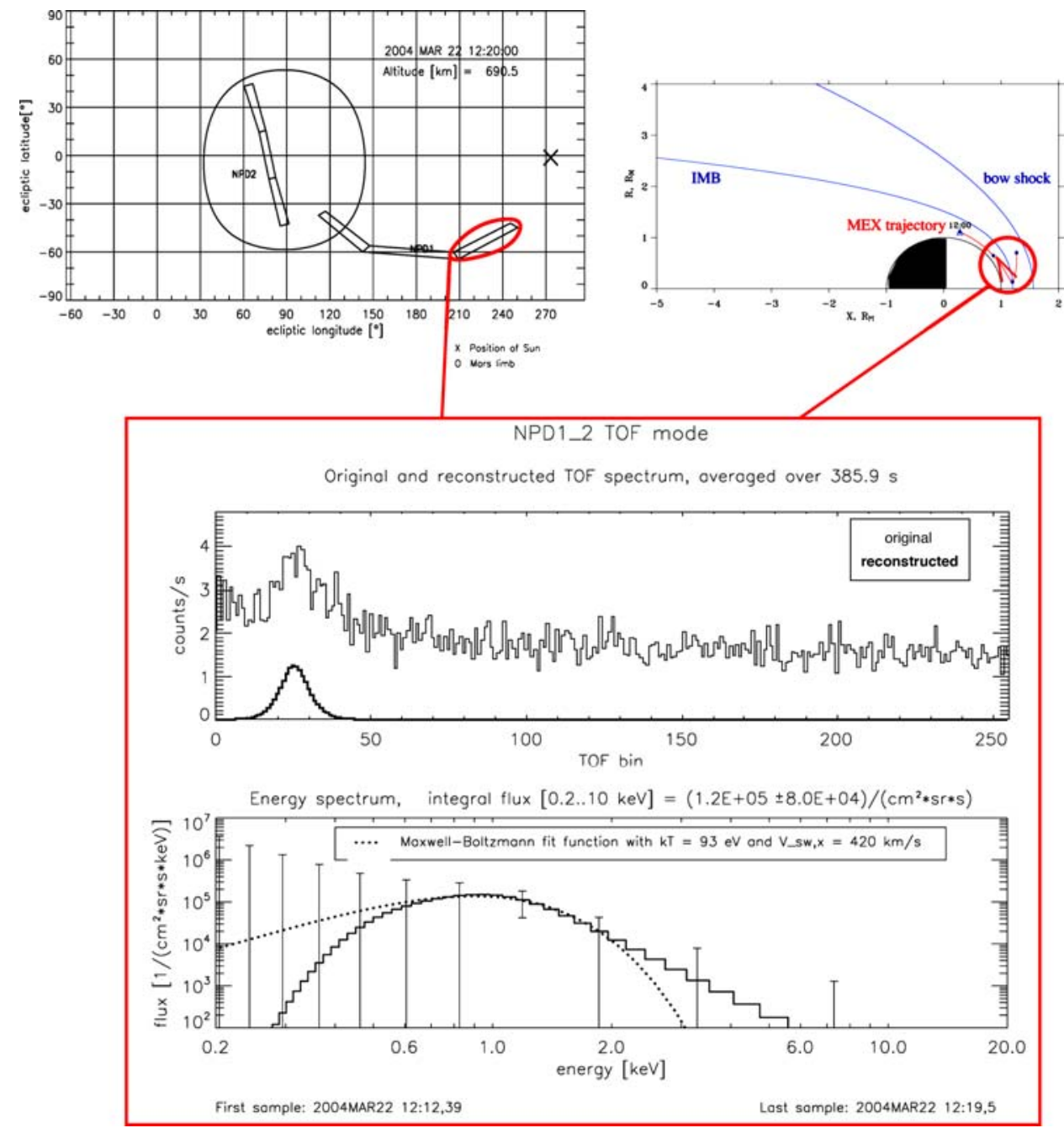

Figure 7. Solar wind ENAs on the dayside of Mars above the subsolar point. The data format is the same as in Figure 3, except for the error bars given for some of the energy bins. This measurement was made when the spacecraft was very close to the Mars surface, near the subsolar point, and the NPD channel 1_2 was pointed to the vicinity of the Sun. The energy spectrum (panel on the bottom) may easily be interpreted as maxwellian distributed solar wind protons that have been neutralized in the Mars hydrogen exosphere. Shortly after the indicated observation period the ENA intensity increased to several $10^{6} \mathrm{~cm}^{-2} \mathrm{sr}^{-1} \mathrm{~s}^{-1}$ and the NPD detector reached saturation.

$\mathrm{s}^{-1}$ for the parent proton distribution. Because of the short integration time and the high UV background level compared to the TOF signal the error bars (plotted in Figure 7 are the 1- $\sigma$ error bars) of the single energy bins are huge. The optimized parameters for $k T$ and $v_{s w, x}$ therefore have to be regarded with caution. 
The integral intensity of $J_{\mathrm{ENA}}=10^{5} \mathrm{~cm}^{-2} \mathrm{sr}^{-1} \mathrm{~s}^{-1}$ in Figure 7 is what one expects for a stream of solar wind protons that are neutralized in the Mars hydrogen exosphere:

$$
J_{\mathrm{ENA}}=\sigma \int_{\mathrm{LOS}} d s n_{\mathrm{H}}(r) J_{\mathrm{p}}=2 \times 10^{5} \mathrm{~cm}^{-2} \mathrm{sr}^{-1} \mathrm{~s}^{-1} .
$$

For $\Sigma_{\mathrm{H}}$ we have chosen $10^{16} \mathrm{~m}^{2}$ according to a UV limb emission measurement in April 2004 (Galli et al., this issue); for the proton beam $J_{\mathrm{p}}=10^{8} \mathrm{~cm}^{-2} \mathrm{sr}^{-1} \mathrm{~s}^{-1}$, assuming a solar wind speed of $420 \mathrm{~km} \mathrm{~s}^{-1}$, and a proton density of $5 \mathrm{~cm}^{-3}$. The thermal spread of the ENA spectrum shown in Figure 7 is somewhat too high for undisturbed solar wind protons and ENAs from undisturbed solar wind are unlikely to scatter in an angle of 60 around the Sun direction. This ENA signal probably is another example of the subsolar ENA jet of shocked solar wind protons, first described by Futaana et al. (2006b).

The typical spectrum of nightside ENAs (Equation (3)) is much broader than the spectrum of dayside solar wind ENAs in Figure 7, and it cannot be parameterized by the Maxwell-Boltzmann distribution of Equation (6). The width of the spectrum rather favors acceleration processes of planetary ions as possible explanation. There are, however, other examples of neutralized solar wind on the dayside whose spectra look similar to the one shown in Figure 3. The energy spectrum cannot be used as evidence against solar wind ENAs because the detected ENA spectrum is a convolution of charge-exchange processes along a LOS that intersects several regions of varying temperature, density and flow directions. Inside the IMB, there is no directed flow of solar wind protons anyway and the simple Equation (5) no longer applies. To our knowledge, there is only the model of Lichtenegger et al. (2002) that separately derives energy spectra of planetary and solar wind ENAs for a few special locations inside the magnetosheath, but these locations cannot be directly compared to our observations. Without further model work we can only assume that the high energy part of the spectrum above $1 \mathrm{keV}$ is due to ENAs that originate from planetary pick-up protons in front of the bow shock but we generally cannot distinguish between the contributions of solar wind protons and of planetary protons to the typical ENA spectrum (Equation (3)). In the following Section 4.3 we will show the only example for which we can actually separate a low-energy and a high-energy ENA component, and in Section 4.4 we will see if we can at least compare the measured integral intensities to theoretical models.

\subsection{Magnetosheath Measurements}

On April 25, 2004, NPD was switched on while the spacecraft was still inside the magnetosheath, far in the downwind region (Figure 8). There, a distinct bimodal spectrum of ENAs abruptly appeared and vanished again half an hour later when the spacecraft was still outside the IMB. Afterwards the intensity decreased 

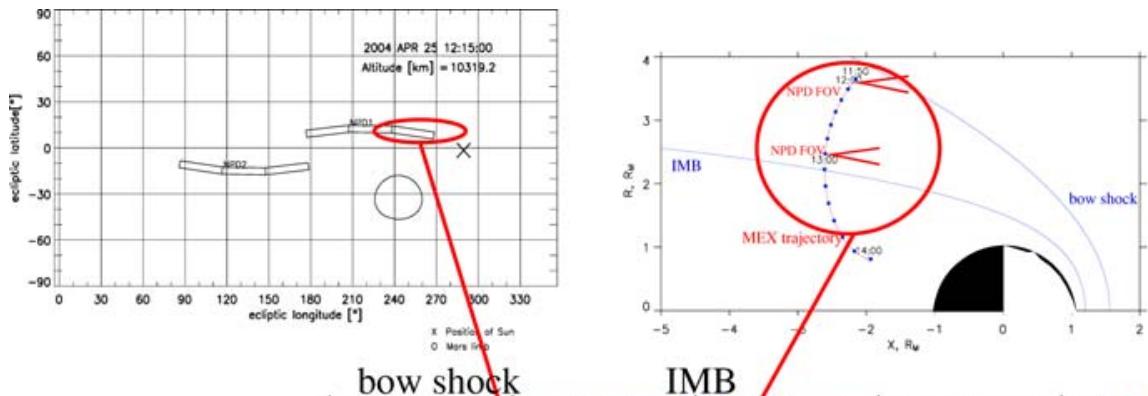

TOF mode
npd1 2
Count rotea $[1 / 8]$
overoged over $32.0 \mathrm{~s}$
from: 2004APR25 11:52,37

until: 2004APR25 14:9,50

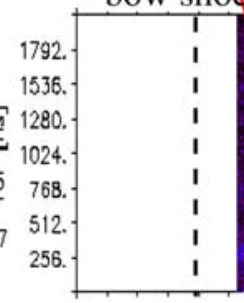

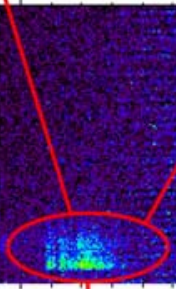
11:00

12:00
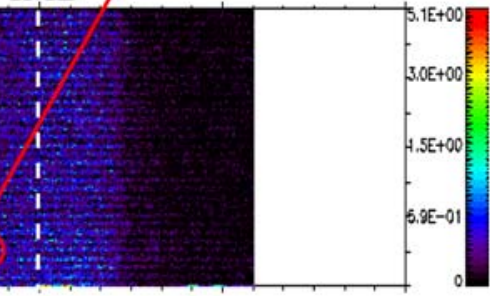

\section{3:00} time [UTC]

$14: 00$

15:00

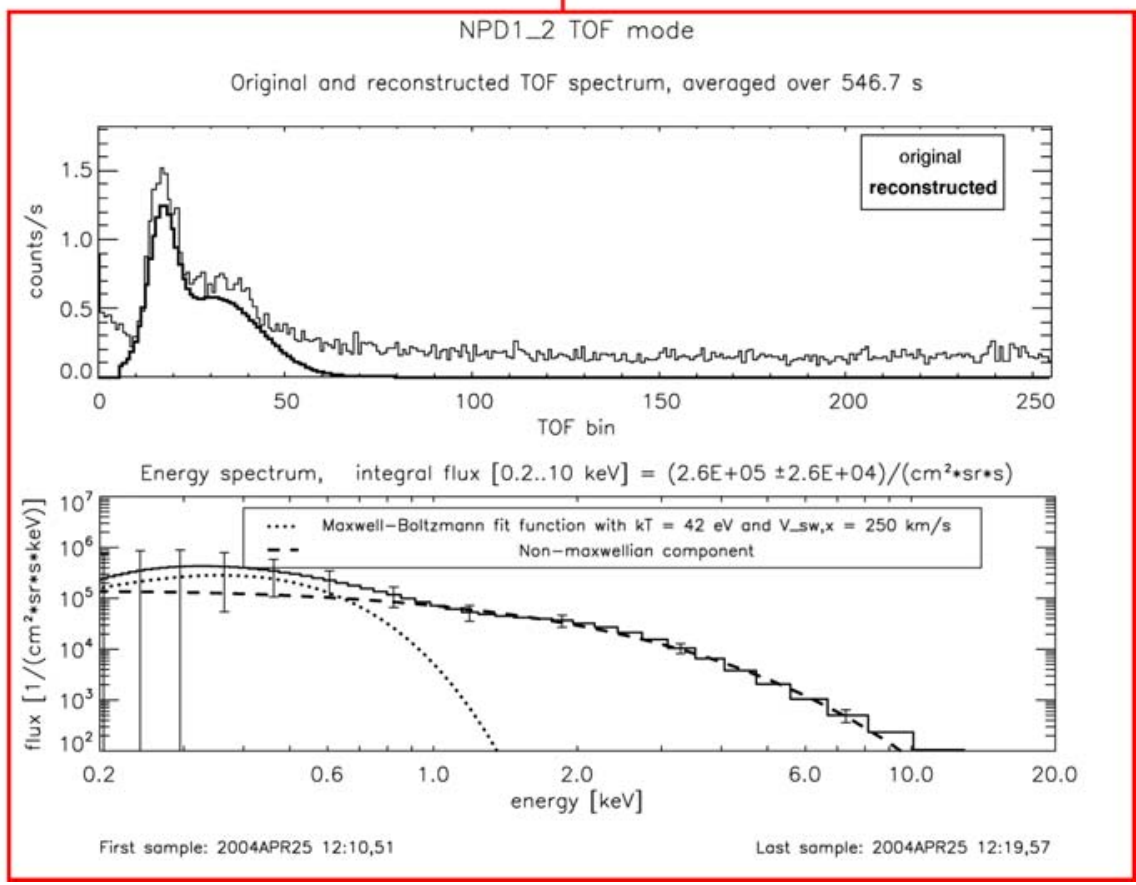

Figure 8. Hydrogen ENAs measured on April 25, 2004. The two uppermost panels show the observation configuration, in the middle follows the color-coded TOF measurement for the entire observation period of $2.25 \mathrm{~h}$. The two lower panels show the TOF signal, averaged over 10 minutes from 12:10 to 12:20 UT, when the intensity reached its maximum. The dashed and the dotted line in the lowermost panel are fit curves for a maxwellian and a two-component power law distribution. The position of the IMB and of the bow shock refer to the plasma model of Kallio et al. (1997). 
below $10^{5} \mathrm{~cm}^{-2} \mathrm{sr}^{-1} \mathrm{~s}^{-1}$, and the usual ENA tail spectrum, as shown in Figures 3 and 6, was measured. Contrary to ion and electron data, the crossing of the IMB is not seen in NPD data, ENA imaging does not allow to map local plasma boundaries.

The ENA signal measured on April 25 is the only example of a bi-modal spectrum in the data base, and it is the only observation made on the nightside of Mars inside the magnetosheath, far away from the IMB. The spectrum shows a peak at 0.35 $\mathrm{keV}$ and a smeared out roll-over at $2.8 \mathrm{keV}$. Neither of the two components can be ENAs originated from undisturbed solar wind; the one with the roll-over at $2.8 \mathrm{keV}$ is much too broad for typical solar wind temperatures, whereas energies of $0.35 \mathrm{keV}$ are much too small for typical solar wind speeds between 400 and $700 \mathrm{~km} \mathrm{~s}^{-1}$. The IMA data of $\mathrm{H}^{+}$and $\mathrm{He}^{2+}$ show that during the NPD measurement the local solar wind bulk velocity in the outer magnetosheath was still $0.9 \mathrm{keV}$ (M. Fraenz, personal communication, 2006). The angle between the field-of-view to the Sun direction is much larger than on March 22 (see Figure 7), and yet the integral intensity again reaches several $10^{5} \mathrm{~cm}^{-2} \mathrm{sr}^{-1} \mathrm{~s}^{-1}$. If we try to parameterize the bi-modal spectrum with the sum of two independent maxwellian distributions according to Equation (6), we find that the peak at $0.35 \mathrm{keV}$ can be reproduced as slowed down, neutralized solar wind protons with a thermal spread of 20 to $200 \mathrm{eV}$ and with a bulk velocity, $v_{s w, x}$, from 0 to $300 \mathrm{~km} \mathrm{~s}^{-1}$. This is the dotted curve in the energy spectrum of Figure 8; the error bars at energies below $0.3 \mathrm{keV}$ make a more accurate estimation impossible. The other component of the energy spectrum in Figure 8, represented by the dashed line, cannot be fitted by a maxwellian distribution with physically meaningful parameters without assuming an unreasonably high temperature. A two-component power law fit see Equation (2) for this component gives a roll-over of $2.8 \pm 0.2 \mathrm{keV}$.

Ion measurements done with IMA (Dubinin et al., 2006a,b,c) have proven the existence of planetary oxygen and hydrogen ions that are accelerated up to several $\mathrm{keV}$ within the magnetosheath. Oxygen ENAs can be excluded because $\mathrm{O}^{+}$ions would have to be accelerated to over $30 \mathrm{keV}$ to reproduce the corresponding peak in the TOF spectrum. We conclude that the ENA component with the roll-over at $2.8 \mathrm{keV}$ is due to planetary pick-up protons, whereas the low-energy component is due to decelerated solar wind protons. This conclusion is confirmed by the model of Lichtenegger et al. (2002) who predict that the planetary ENAs created upstream of the bow shock and in the outer magnetosheath have higher energies than the solar wind ENAs. The ratio of the energies of the two components found in Figure 8 $(2.8 \mathrm{keV} / 0.35 \mathrm{keV})$ is higher than the factor of 4 we would expect if the stream of slow solar wind protons that produced the low energy peak had been the place where the high energetic pick-up ions were accelerated (Dubinin et al., 2006c). The reason is that the NPD field-of-view did not cover the subsolar point, missing the streamlines of the fast solar wind. NPD detected only ENAs from the hot, decelerated solar wind protons and the planetary pick-up ENAs, which have a broader angular distribution than the solar wind itself. The integral intensity of 
the high-energy component, on the other hand, is astonishingly high: Lichtenegger et al. (2002) predict that the planetary H-ENA flux reaches at most $20 \%$ of the solar wind ENA flux, since the charge-exchange between solar wind protons and hydrogen neutrals is an important source for the planetary $\mathrm{H}^{+}$that subsequently create planetary H-ENAs. In contrast, we find for the spectrum in Figure 8 that the broad component with a roll-over at $2.8 \mathrm{keV}$ accounts for as much as $60 \%$ of the entire ENA intensity $J_{\mathrm{ENA}}$ between 0.2 and $10 \mathrm{keV}$. To produce a beam of planetary H-ENA with an intensity of $2 \times 10^{5} \mathrm{~cm}^{-2} \mathrm{sr}^{-1} \mathrm{~s}^{-1}$ it takes (according to Equation 7) a stream of planetary protons of $10^{8} \mathrm{~cm}^{-2} \mathrm{sr}^{-1} \mathrm{~s}^{-1}$ at some place in the magnetosheath. Up to now, no such intense pick-up proton streams with sufficient energies have been found in IMA data (Dubinin et al., 2006c).

The energy spectrum and the integral intensity of the broad component are similar to the typical spectrum of tailward ENAs seen inside the IMB except for the place of the roll-over. For any other measurement in the data base the roll-over lies at energies well below $2 \mathrm{keV}$ (Figure 5). We do not have other measurements from the magnetosheath on the nightside to assess if April 25, 2004, was a singular case or if ENAs measured in the outer magnetosheath always would show a bi-modal distribution.

\subsection{Flux Statistics}

The integrated ENA intensities can be organized into maps to be compared with simulation results. We combine all those measurements where the solar zenith angle and the distance to Mars are similar to one single plot. All measurements of tailward flowing ENAs were taken into account, including those weak ENA streams whose energy spectra are poorly constrained. During the NPD observations (see Figure 2) the solar zenith angle varied only between $135^{\circ}$ and $160^{\circ}$. Because the orbit and the operational phase did not evolve much from April to May 2004 the entire data set (with the exception of the three observations in February) of 10 hours of NPD measurements may be presented by just four maps at different distances from the planet; they are shown in Figure 9. The top left panel (a) illustrates the measurement on April 25, 2004, in the magnetosheath (see Section 4.3), the other three panels (b, c, d) show the integral intensities measured inside the IMB (discussed in Section 4.1 ) at distances of 2.0, 1.0, and $0.6 R_{\mathrm{M}}$. We observe a homogenous picture of integral intensities inside the IMB. The signals from the Mars disk itself are weak, bordering to the detection limit of $10^{4} \mathrm{~cm}^{-2} \mathrm{sr}^{-1} \mathrm{~s}^{-1}$, the more intense signals are seen around the limb close to the Sun direction. They reach values of a few $10^{5}$ $\mathrm{cm}^{-2} \mathrm{sr}^{-1} \mathrm{~s}^{-1}$ at most, corresponding to the yellow areas in Figure 9.

To map the global production rate of ENAs it would be desirable to combine the H-ENA signals that were observed close to the Sun direction in an additional image as one expects the maximum intensity of tailward ENAs to be parallel to the proton streamlines. The images in Figure 9 show all available data but they include 


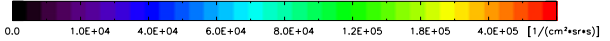

a)
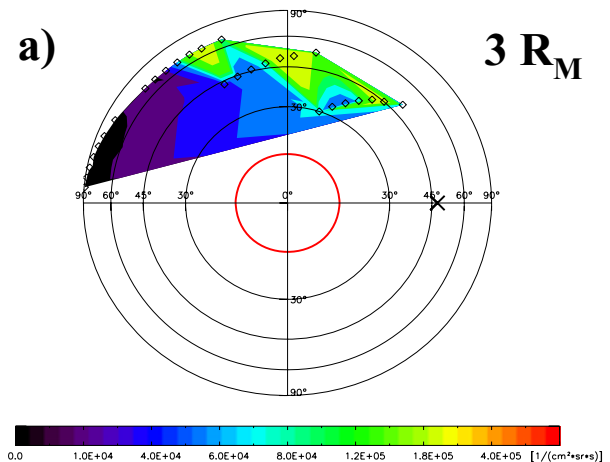

c)

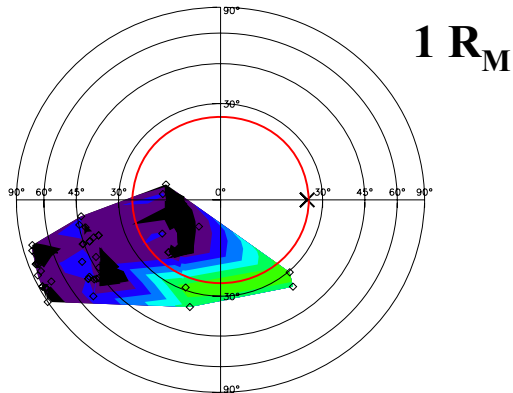

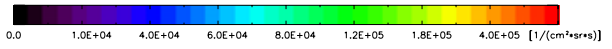

b)

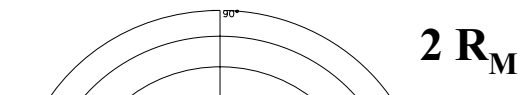

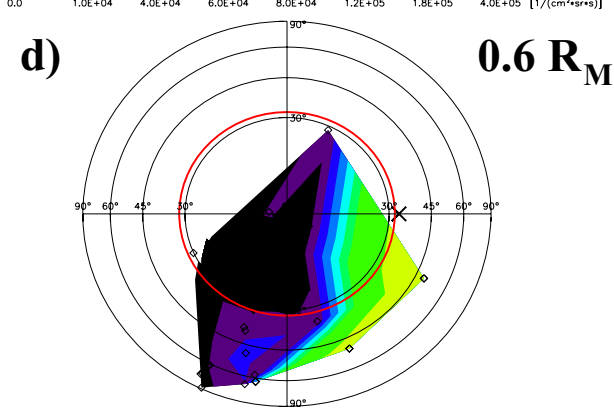

Figure 9. Images of integral H-ENA intensities in polar coordinates as seen from MEX. The spacecraft follows the trajectory shown in Figure 2, the distance to the Mars surface (red bold circle) decreases from $3 R_{\mathrm{M}}$ (a) over $2 R_{\mathrm{M}}$ (b) and $1 R_{\mathrm{M}}$ (c) to about $0.6 R_{\mathrm{M}}$ (d). The solar zenith angle is roughly $150^{\circ}$ for all plots ( $\mathrm{X}$ indicates the Sun position). The tiny diamonds denote the boresight directions of the NPD channels during the single observations. Image $9 \mathrm{~d}$ can directly be compared to the model prediction shown in Figure 10.

directionality effects since the angle between the NPD field-of-view and the Sun varies from $30^{\circ}$ to $120^{\circ}$. The ENA intensity drops quickly as the field-of-view is directed away from the Sun. Unfortunately, there are only two data points of 10 minutes each for which the angle between the NPD field-of-view and the Sun is smaller than $30^{\circ}$ (the yellow area in Figure 9d). The reason for evading the Sun is the UV sensitivity of NPD. To estimate the upper limit of the global ENA production rate in Section 6 we will assume that the ENA intensities of $2 \times 10^{5} \mathrm{~cm}^{-2} \mathrm{sr}^{-1} \mathrm{~s}^{-1}$ measured at these occasions apply to observations all around the Mars limb as long as the field-of-view is directed to the sub-solar point.

As comparison to the measurements shown in Figure 9d, Figure 10 shows the predictions for solar wind ENAs of a model from Gunell et al. (2006), and Figure 11 shows the predictions for planetary ENAs of the model from Lichtenegger et al. (2002), which has to be compared to Figure 9a. The ENA image shown in Figure 10 has been calculated for the observation configuration of the ENA measurements shown in Figure 9d. In the published version (Gunell et al., 2006) 


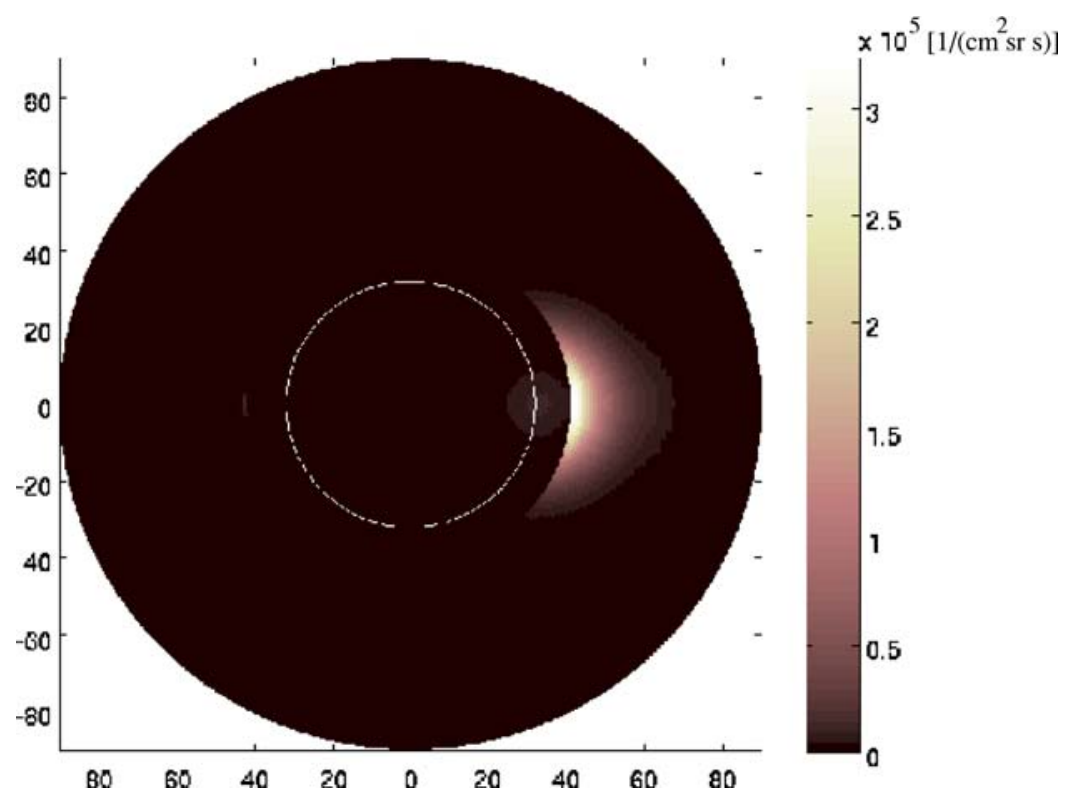

Figure 10. Model of solar wind ENA intensities (Gunell et al., 2006) that has been adapted to the NPD measurement conditions in Figure 9d. The modeled solar wind ENA intensity increases to a few $10^{5} \mathrm{~cm}^{-2} \mathrm{sr}^{-1} \mathrm{~s}^{-1}$ close to the Sun direction. The axes are plotted in polar coordinates in units of degrees.

had taken the hydrogen exosphere parameters from the model of Krasnopolsky and Gladstone (1996). For the calculation presented here the values for the exobase density and temperature were replaced by recent measurements (Galli et al., this issue). Krasnopolsky and Gladstone (1996) predicted for low solar activity $n_{\mathrm{H}}=10^{12} \mathrm{~m}^{-3}$ for the hydrogen exobase density and $T=200 \mathrm{~K}$ for the temperature. However, the UV Lyman- $\alpha$ limb emission (Galli et al., this issue) measured during the orbit of April 25, 2004, indicates a much thinner hydrogen exosphere, $n_{\mathrm{H}}=10^{10} \mathrm{~m}^{-3}$, with a very hot component above the exobase, $T \geq 600 \mathrm{~K}$. The hydrogen column densities are an order of magnitude lower than predicted by the model (Krasnopolsky and Gladstone, 1996) and the ENA intensities are bound to be lower than formerly calculated. The spatial distribution and the integral intensity of the solar wind ENA model (Figure 10) now match the observations (Figure 9d), whereas the cool and dense hydrogen exosphere model used in the published version (Gunell et al., 2006) leads to H-ENA intensities that are an order of magnitude too high.

The planetary ENA fluxes shown in Figure 11, on the other hand, are taken directly from the publication of Lichtenegger et al. (2002) who also applied the model values published by Krasnopolsky and Gladstone (1996). This might explain why the measured H-ENA intensities are one or two orders of magnitude lower than Figure 11 suggests. If we assume that the high energetic H-ENA component seen 

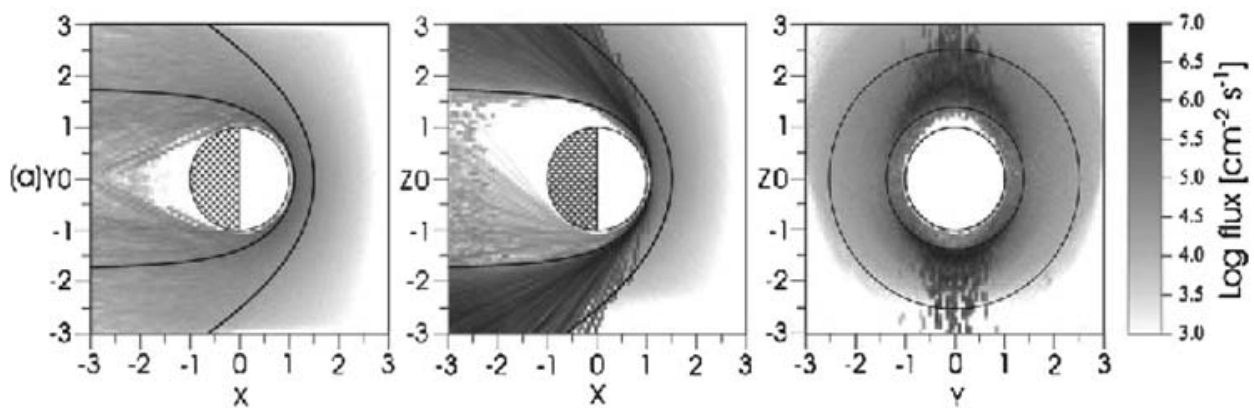

Figure 11. Modeled planetary ENA fluxes for solar minimum, taken from the work of Lichtenegger et al. (2002). The regions of intense production of planetary ENAs (black areas) are predicted to be aligned with the solar wind electric field ( $Z$-axis in their reference frame). There, the modeled ENA flux exceeds $10^{6} \mathrm{~cm}^{-2} \mathrm{~s}^{-1}$, whereas NPD observed only $10^{5} \mathrm{~cm}^{-2} \mathrm{~s}^{-1}$ (see Figure 9a and Equation (8)).

in the magnetosheath (Figure 9a) is due to planetary hydrogen atoms only we find for the measured ENA flux $F_{p l}$ of planetary hydrogen atoms at most

$$
F_{p l} \leq 2 \times 10^{5} \mathrm{~cm}^{-2} \mathrm{~s}^{-1} \mathrm{sr}^{-1} \times 0.5 \mathrm{sr}=10^{5} \mathrm{~cm}^{-2} \mathrm{~s}^{-1},
$$

where we have treated the ENA signal as a beam cone of roughly $0.5 \mathrm{sr}$ aperture. This is about one order of magnitude below the predicted maximum of $10^{6} \mathrm{~cm}^{-2} \mathrm{~s}^{-1}$ for the tailward H-ENA fluxes in Figure 11 calculated by Lichtenegger et al. (2002).

This comparison can only be qualitative because at every spacecraft position measurements of incoming ENAs from all directions (solid angle of $4 \pi \mathrm{sr}$ ) would be required to transform the measured ENA intensities into a map of integral fluxes as presented in Figure 11. Equation (8) gives a valid estimate if the detected stream of ENAs is much more intense than ENA signals from any other direction at that place in the magnetosheath. According to the model of Lichtenegger et al. (2002) this assumption is justified: The dark region of maximum outflow in Figure 11 is aligned with the solar wind electric field. In this picture planetary ENAs originate from ionized hydrogen atoms that have been accelerated in the electromagnetic field of the solar wind outside the IMB before undergoing charge-exchange. MEX lacks a magnetometer but the proxies for the IMF direction derived from MGS data (2006) indicate that the magnetic field of the solar wind was directed dawnward on April 25, 2004. We can therefore assume that the measured maximum of ENA outflow seen above Mars (Figure 9a) really is coaligned with the direction of the electric field $\mathbf{E}=-\mathbf{v} \times \mathbf{B}$ at the given date and that this ENA hot spot corresponds to one of the two dark spots of planetary ENAs in Figure 11. We cannot exclude, however, that the IMF direction varied on a timescale smaller than the two hour interval provided by MGS magnetometer measurements (Brain, 2006). Although we are not certain about the IMF direction we establish a discrepancy between measured and predicted fluxes. The model of Lichtenegger et al. (2002) predicts 
solar wind ENA fluxes around the Mars limb that are even higher than the planetary ENAs by a factor of five.

Except for the one measurement in the magnetosheath (Section 4.3) the presented H-ENA signals can be understood as solar wind ENAs. The energy spectra do not allow us to deduce the source of the observed ENA signals measured inside the IMB. However, a solar wind generated ENA stream is expected to be strongly concentrated around the Mars limb in the Sun direction (see Figure 10), whereas a planetary ENA signal should be aligned with the electric field of the solar wind (see Figure 11). We do not know the exact direction of this field during the observations, but within the time span of roughly three weeks that covers the ENA images in Figure 9 we expect it to be varying randomly in the plain perpendicular to the Sun direction. It is therefore unlikely that regions with potentially larger ENA production always escaped our attention. The only region where we find high ENA intensities around the Mars limb is correlated with the direction to the Sun. This is the strongest argument that the majority of the H-ENAs seen inside the IMB are due to solar wind protons, and the intensity of a few $10^{5} \mathrm{~cm}^{-2} \mathrm{sr}^{-1} \mathrm{~s}^{-1}$ is consistent with solar wind ENAs, too. The measured ENA image fits to the predicted image of solar wind ENAs (compare Figures 9d and 10) except for the ENA streams from the planetary surface itself. These signals either are due to planetary ENAs, or the sharp obstacle boundary that is impenetrable to solar wind ENAs in the model is an oversimplification. According to calculations done by Kallio et al. (2006) solar wind ENAs are expected to spread into the eclipse because of the thermal spread of the parent solar wind protons and because the ENAs are scattered in the martian exosphere. Closer than $2 R_{\mathrm{M}}$ above the planet in deep eclipse this signal of solar wind ENAs should be orders of magnitude lower than seen at Mars limb (Kallio et al., 2006). The NPD measurements do not allow us to test this prediction as the spacecraft was never in deep eclipse.

Because the intensity as well as the spatial distribution of the ENA signals are consistent with model predictions for solar wind ENAs (Figure 10) we conclude that the majority of the ENAs measured inside the IMB are due to solar wind protons. Model calculations (Lichtenegger et al., 2002) also indicate that the ratio of planetary to solar wind ENA fluxes should not exceed $20 \%$ at any time of the solar cycle. We cannot exclude that we have overlooked a hot spot of ENA production on the nightside of Mars (such as the dark region shown in the model plot in Figure 11) because our database is limited to a few months in 2004 and the pointing directions do not fully cover the magnetosheath or even the IMB. The narrow field-of-view covers only $6\left(30^{\circ} \times 4^{\circ}\right)$ of the entire sphere, which makes a comparison to modeled ENA fluxes difficult. Moreover, the electromagnetic configuration of the solar wind is not known either for most measurements. Nonetheless, the various observations on different days show a homogeneous picture of the tailward ENA flow. Inside the IMB the only hot spot of ENA production is related to the Sun direction; in the magnetosheath the burst of ENAs coincides with the predicted hot spot of planetary ENA production. For the following discussions we shall assume that the NPD ENA 
maps give us a representative image of the ENA flow at the martian nightside for solar minimum conditions and that no ENA hot spot has been overlooked.

\section{Where are the Oxygen ENAs?}

In analogy to hydrogen ENAs simulations predict significant oxygen ENAs in the tailward flow. Figure 13 shows the differential intensity of tailward O-ENAs according to the model of Barabash et al. (2002) for solar zenith angles $135^{\circ}$ and $180^{\circ}$. The maximum energy to which an oxygen ion can be accelerated within the IMB region before charge-exchanging with neutral hydrogen or oxygen is predicted to be $1.7 \mathrm{keV}$. The bright spot of O-ENAs in the right panel, e.g., corresponds to an integral intensity of $J_{\mathrm{ENA}} \approx 10^{6} \mathrm{~cm}^{-2} \mathrm{sr}^{-1} \mathrm{~s}^{-1}$ for the energy range between 0.1 and $1 \mathrm{keV}$. On the other hand, Gunell et al. (2006) compare the results of an empirical, a hybrid, and an MHD model; depending on the model they find maximum O-ENA intensities varying between $10^{4}$ and $10^{6} \mathrm{~cm}^{-2} \mathrm{sr}^{-1} \mathrm{~s}^{-1}$ for the same observation conditions as plotted in Figure 13. These O-ENA simulations obviously are modeldependent. The exosphere parameters used in all models of Barabash et al. (2002) and Gunell et al. (2006) are taken from Krasnopolsky and Gladstone (1996).

O-ENA intensities as high as predicted by Barabash et al. (2002) should easily be detected by NPD, which has a detection limit of about $10^{4} \mathrm{~cm}^{-2} \mathrm{sr}^{-1} \mathrm{~s}^{-1}$ for neutral oxygen between 0.3 to $10 \mathrm{keV}$. Figure 12 shows the NPD instrument response to a monoenergetic oxygen beam of $0.7 \mathrm{keV}$ from the calibration. The signature is flatter than for hydrogen ENAs but it is still well recognizable in the TOF spectrum. Because of the higher atomic mass, the peak in the TOF spectrum lies at much higher bins than for hydrogen ENAs of comparable energies (see for instance the TOF signal of neutralized solar wind protons in Figure 7).

In contrast to theoretical predictions there is not a single occasion in the entire data set of 10 hours where an oxygen ENA signal is unambiguously detected. In

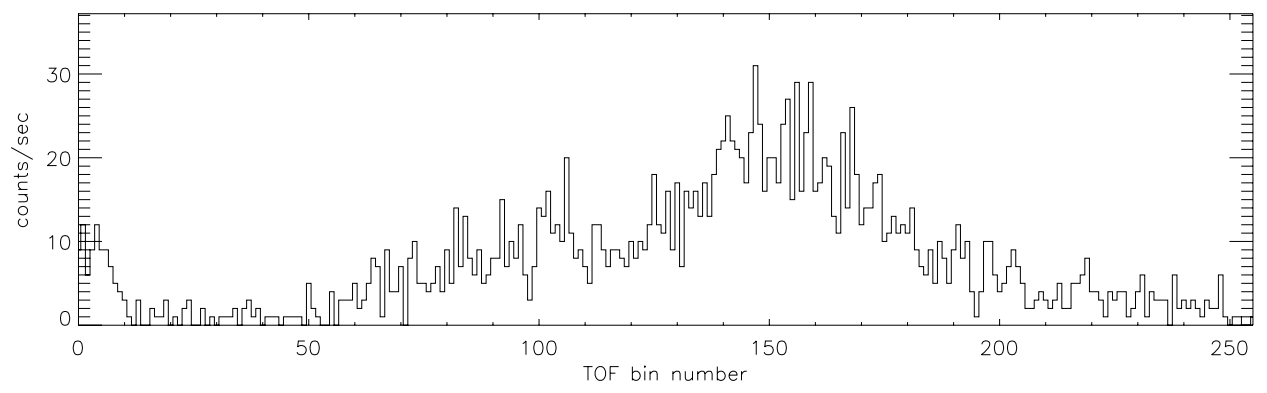

Figure 12. Response of the NPD2_1 channel to a beam of monoenergetic oxygen ENAs of $0.7 \mathrm{keV}$ from the calibration. Incoming O-ENAs produce a peak in the TOF spectrum at higher TOF bins than H-ENAs and can thus be distinguished. 

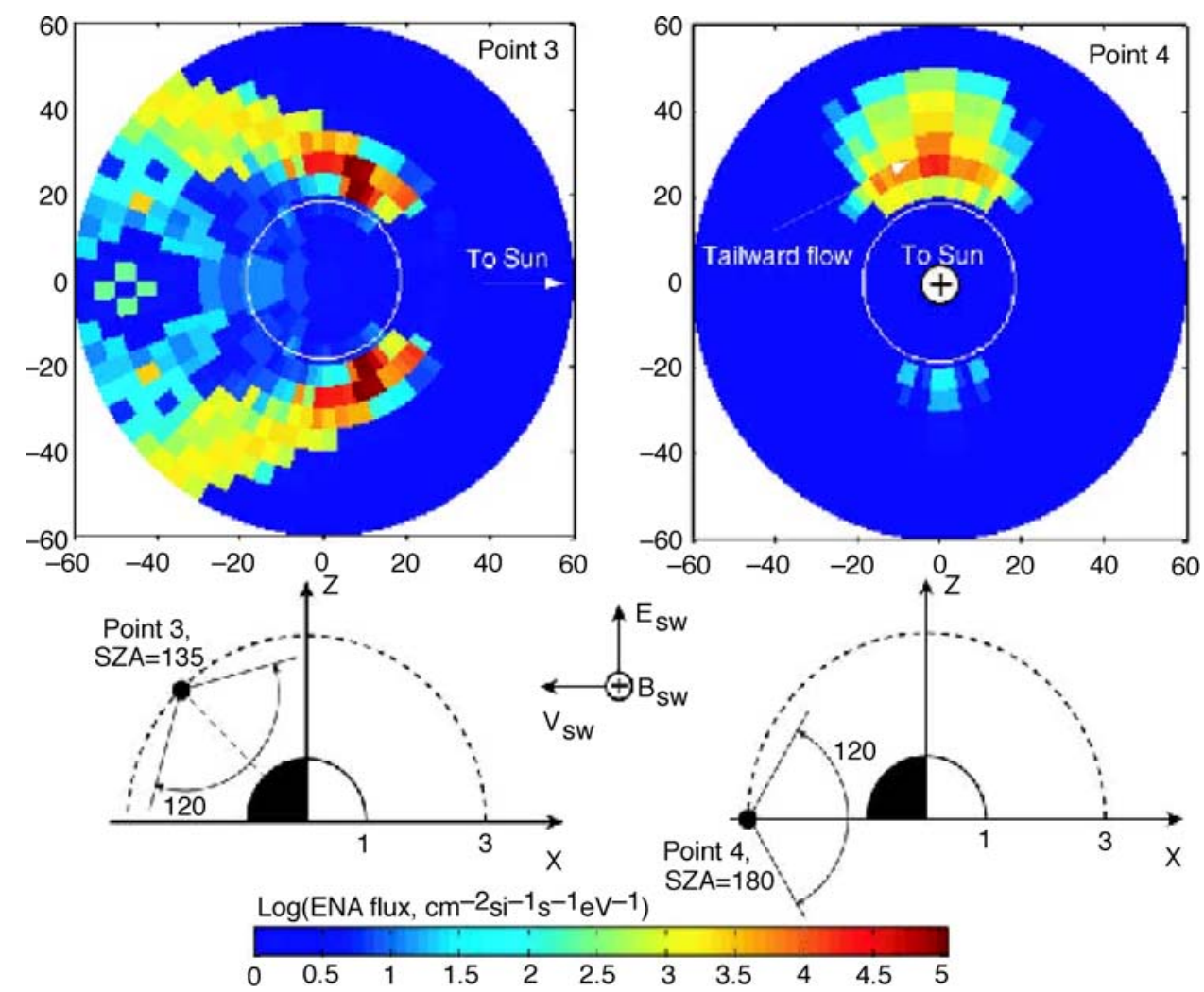

Figure 13. Model predictions for the oxygen ENA intensity on the nightside of Mars, taken from Barabash et al. (2002). Note that the intensity is given in $\mathrm{eV}^{-1}$ instead of $\mathrm{keV}^{-1}$ ! In the right panel the ENA stream concentrates to the direction parallel to the electric field of the solar wind. Planetary oxygen ions are convected and accelerated to the same direction as planetary hydrogen atoms are (see right top panel of Figure 11).

most cases the TOF spectrum shows only UV photon noise in the TOF bins from 100 to 250 that would correspond to O-ENAs, and when there is a peak in the spectrum it is too flat to be significant. Even if we assume for all measured TOF spectra that all counts above the photon noise level in the bins from 100 to 250 were produced by O-ENAs the integral intensity never exceeds $J_{\mathrm{ENA}}=2 \times 10^{4} \mathrm{~cm}^{-2}$ $\mathrm{sr}^{-1} \mathrm{~s}^{-1}$, integrated from 0.4 to $1 \mathrm{keV}$.

In summary, model calculations predict for comparable observation conditions oxygen ENA intensities that are one or two orders of magnitude higher than the upper limit of $10^{4} \mathrm{~cm}^{-2} \mathrm{sr}^{-1} \mathrm{~s}^{-1}$ of potential O-ENAs that we find in spring 2004. As we already stated in the discussion of the H-ENA integral fluxes (Section 4.4) it is improbable that an ENA hot spot as shown in Figure 13 was not discovered because the electric field of the solar wind was perpendicular to the NPD field-of-view for every observation. It is more plausible that O-ENA streams above the detection 
limit do not exist for solar minimum conditions. This is understandable because in 2004 the neutral hydrogen exosphere is much thinner than previously thought: Barabash et al. (2002) also used the neutral density profiles from Krasnopolsky and Gladstone (1996), which predict a hydrogen exobase density two decades higher than actual measurements for solar minimum (Galli et al., this issue).

There is increasing evidence from IMA that also the Mars ionosphere was thinner during the MEX mission than predicted by models. Dubinin et al. (2006b) show that oxygen ions accelerated to several $\mathrm{keV}$ inside the IMB do exist, and a recent evaluation of IMA data (Barabash et al., 2006) indicates that the integral flux of $\mathrm{O}^{+}$and $\mathrm{O}_{2}^{+}$is two orders of magnitude lower than the previous estimate from 1989 derived by Lundin et al. (1989) from Phobos 2 measurements. The O-ENA model of Barabash et al. (2002) was scaled to reproduce a global oxygen ion loss rate of $10^{25} \mathrm{~s}^{-1}$, whereas the integration of $\mathrm{O}^{+}$fluxes, measured with IMA at solar minimum from 2004 to 2006 , leads to a total ionospheric loss rate of only $10^{23} \mathrm{~s}^{-1}$. The oxygen ion intensities inside the IMB are found to reach $J_{\mathrm{p}}=10^{6} \mathrm{~cm}^{-2} \mathrm{sr}^{-1} \mathrm{~s}^{-1}$ at most (Barabash et al., 2006). We lack recent measurements of the neutral oxygen exosphere to predict O-ENA intensities from these ion intensities. According to recent models (Krasnopolsky, 2002; Lichtenegger et al., this issue) the radial column density of neutral oxygen, $\Sigma_{\mathrm{O}}$, is less than $4 \times 10^{15} \mathrm{~m}^{-2}$ for altitudes above $600 \mathrm{~km}$. Since planetary oxygen ions typically reach energies $\geq 0.5 \mathrm{keV}$ only above 1000 $\mathrm{km}$ (Dubinin et al., 2006b), the production of O-ENAs is therefore dominated by the neutral hydrogen exosphere. We expect:

$$
J_{\mathrm{ENA}} \approx \sigma \Sigma_{H} J_{\mathrm{p}}(s)=10^{3} \mathrm{~cm}^{-2} \mathrm{sr}^{-1} \mathrm{~s}^{-1},
$$

with the cross section $\sigma=10^{-15} \mathrm{~cm}^{2}$ for the charge-exchange between $\mathrm{O}^{+}$and $\mathrm{H}$ (Figure 1) and a hydrogen column density of $\Sigma_{H}=10^{16} \mathrm{~m}^{-2}$. Obviously, the nonexistence of oxygen ENA signals above the NPD detection threshold is consistent with the measured $\mathrm{O}^{+}$fluxes and the neutral hydrogen exosphere that are both one or two orders of magnitude thinner than assumed in the models of Barabash et al. (2002) and Gunell et al. (2006). The NPD observations are consistent with recent oxygen exospheric models (Krasnopolsky, 2002; Lichtenegger et al., this issue), but do not rule out that the oxygen exosphere was also thinner in 2004 than theoretically predicted.

\section{Global ENA Production Rates of Hydrogen and Oxygen}

There are two problems if we try to estimate the global production rate of hydrogen and oxygen ENAs. First there are not enough measurements to cover the entire magnetosheath. Second, we have not clearly identified any O-ENAs at all, whereas for H-ENAs the ratio between planetary and solar wind ENAs remains unclear. At least we can give upper estimates of the global production rates of ENAs. 
First we assume that the planetary ENA fluxes outside the magnetosheath are negligible. This assumption is justified because outside the magnetosheath only the subsolar ENA jet can contain notable fluxes of planetary ENAs. Futaana et al. (2006b) find that the integral intensity of the ENA jet amounts to only $5 \times 10^{5} \mathrm{~cm}^{-2}$ $\mathrm{sr}^{-1} \mathrm{~s}^{-1}$, including solar wind and planetary ENAs. Because the source region of this jet is typically not more than a few $100 \mathrm{~km}$ in diameters the resulting loss rate amounts to $10^{20} \mathrm{~s}^{-1}$ at most. Second, we assume that no ENA hot spot has escaped our observations. This is plausible, but for future planetary ENA imaging missions it will be important to better map the magnetosheath as the ENA flows in the magnetosheath dominate the estimates of global production rates. Third, we integrate the H-ENA intensities shown in Figure 9 over aperture angle and cross section of the IMB and of the bow shock at the terminator. For this estimation we have approximated the IMB and the bow shock shape as axis-symmetric cones with a constant aperture angle of $26^{\circ}$ for the IMB cone and $52^{\circ}$ for the bow shock cone (Kallio et al., 1997). If all H-ENA signals were entirely due to planetary hydrogen we then obtain $4 \times 10^{23} \mathrm{~s}^{-1}$ as an upper limit of the global production rate of planetary ENAs. For this estimate we have assumed (see Section 4.4) that the tailward flow inside the IMB reaches $2 \times 10^{5} \mathrm{~cm}^{-2} \mathrm{sr}^{-1} \mathrm{~s}^{-1}$ everywhere around the Mars limb for viewing directions close to the Sun and that the high ENA intensities of $3 \times 10^{5} \mathrm{~cm}^{-2} \mathrm{sr}^{-1} \mathrm{~s}^{-1}$ in the magnetosheath (Figure 9a) are restricted to the two spots shown in Figure 11. If the higher ENA intensities around the Mars limb in Figure 9 are due to solar wind protons and only the weak signals from the Mars surface are due to planetary ions the global production rate reduces by an order of magnitude. In summary, we estimate the global production rate of planetary hydrogen ENAs to range between

$$
Q=10^{22} \ldots 4 \times 10^{23} \mathrm{~s}^{-1} .
$$

This is more than a factor of 10 below the estimate of Lichtenegger et al. (2002) of $Q=10^{24} \ldots 10^{25} \mathrm{~s}^{-1}$. It is also less than the production rate of solar wind ENAs on the dayside of Mars. For the ENA dayside albedo, reported by Futaana et al. (2006a), we find with $J_{\mathrm{ENA}}=10^{6} \mathrm{~cm}^{-2} \mathrm{sr}^{-1} \mathrm{~s}^{-1}$

$$
Q=J_{\mathrm{ENA}} \pi R_{M}^{2} 2 \pi \approx 2 \times 10^{24} \mathrm{~s}^{-1}
$$

for the entire hemisphere. The tailward flowing ENAs, be they planetary or not, are a rather inconspicuous feature of the solar wind interaction with the martian atmosphere. The total H-ENA production rate of Mars, integrated over both hemispheres, including solar wind and planetary ENAs, thus amounts to

$$
2 \ldots 3 \times 10^{24} \mathrm{~s}^{-1},
$$

which again is a factor of $10 \ldots 30$ times smaller than predicted by the three models published in Gunell et al. (2006) who apply the exospheric model of Krasnopolsky and Gladstone (1996). 
Since the intensity of oxygen ENAs is at least an order of magnitude less than the intensity of H-ENAs we can set an upper limit of $10^{22} \mathrm{~s}^{-1}$ for the global loss rate of oxygen atoms from the atmosphere due to ENA production. Both for planetary hydrogen and oxygen ENAs the global production rates correspond to atmospheric loss rates of less than $1 \mathrm{~g} \mathrm{~s}^{-1}$.

\section{Conclusions}

1. ENA measurements are a viable means to get a global image of the solar wind interaction with a planetary atmosphere, but to interpret the image theoretical models and knowledge of the exospheric density profile and of the solar wind parameters are mandatory. For future ENA imaging experiments we advise to make more observations in the magnetosheath. The ENA fluxes in the magnetosheath dominate the global ENA loss rate due to the large dimensions of the magnetosheath compared to the space inside the IMB or to the size of the planet. We have also learned that ENA images obtained from inside the IMB are difficult to interpret. The ENA data from the subsequent ASPERA-4/NPD experiment in Venus orbit are expected to be easier to interpret since the Venus Express spacecraft has a magnetometer.

2. On the nightside of Mars hydrogen ENAs up to a few $10^{5} \mathrm{~cm}^{-2} \mathrm{sr}^{-1} \mathrm{~s}^{-1}$ have been measured in the tailward flow, but no oxygen ENA signals have been detected.

It remains unclear to which extent planetary hydrogen contributes to the measured H-ENA intensities. The presence of solar wind ENAs is clearly seen at the dayside (Futaana et al., 2006a, b). On the nightside where the observation configuration should be more favorable to detect planetary ENAs (Lichtenegger et al., 2002) the typical energy spectrum shows only a weak roll-over and does not allow the discrimination of two different populations. It is possible that the weak H-ENA signals from the Mars disk are due to planetary protons (Lichtenegger et al., 2002), the more intense ENA streams of a few $10^{5} \mathrm{~cm}^{-2} \mathrm{sr}^{-1} \mathrm{~s}^{-1}$ close to the Sun direction probably are neutralized solar wind protons. The bimodal distribution seen on April 25 in the magnetosheath looks promising, the high energetic component probably consisting of planetary ENAs, but this is the only case where we can clearly identify two different components in the energy spectrum.

3. The spatial distribution of tailward flowing H-ENAs around the Mars limb is consistent with the solar wind ENA model of Gunell et al. (2006), when modified for a much thinner and hotter hydrogen exosphere. Likewise, the planetary H-ENA signal seen on April 25, 2004, matches the model of Lichtenegger et al. (2002), except that the measured intensities are an order of magnitude lower than predicted. The maximum intensities of hydrogen and oxygen ENAs have been found to be generally one or two decades lower than predicted by 
all model calculations (Barabash et al., 2002; Gunell et al., 2006; Holmström et al., 2002; Kallio et al., 1997; Lichtenegger et al., 2002) that use parameters for the martian hydrogen exosphere from the model of Krasnopolsky and Gladstone (1996). There, a very dense neutral hydrogen exosphere is predicted for solar minimum conditions whilst NPD observations of the Lyman- $\alpha$ airglow suggest a hydrogen surface density that is 20 times lower (Galli et al., this issue). Since the predicted ENA intensity directly depends upon the density of neutral atoms along the LOS, the lower exospheric densities could explain the discrepancy both for hydrogen and oxygen ENAs. The exospheric densities of other species, such as $\mathrm{O}$, influence the production of pick-up ions, but they are of minor importance (Kallio et al., 1997) for the production of hydrogen and oxygen ENAs because of the low scale height of the oxygen corona. The neutral hydrogen density exceeds the neutral oxygen density for altitudes above $500 \mathrm{~km}$ (Lichtenegger et al., this issue) where charge-exchange reactions of the previously accelerated oxygen ions need to take place to produce O-ENAs.

4. To whatever extent planetary atoms have contributed to the measured ENA signals, the derived global production rates correspond to atmospheric loss rates of less than $1 \mathrm{~g} \mathrm{~s}^{-1}$, both for hydrogen and oxygen. This is much less than the total atmospheric loss rate of $1 \mathrm{~kg} / \mathrm{s}$ for hydrogen and for oxygen according to Lammer et al. (2005).

5. Ionospheric escape and dissociative recombination induced escape were thought to be the dominating loss processes for atmospheric oxygen on Mars (for an overview see the work of Chassefière and Leblanc (2004)). An oxygen ion loss rate of $10^{25} \mathrm{~s}^{-1}$ has been estimated from measurements during the Phobos 2 mission in 1989 (Lundin et al., 1989). Kim et al. (1998) and Lammer et al. (2005) estimate that the global oxygen loss rate due to dissociative recombination reaches $10^{25} \mathrm{~s}^{-1}$ as well. At least the ionospheric loss rate needs to be revised in the light of the recent NPD and IMA observations. The missing evidence of O-ENAs alone would not yet urge us to suggest a lower oxygen ion loss rate, but parallel to this work IMA data from 2004 to 2006 have been examined: Barabash et al. (2006) find that at solar minimum the $\mathrm{O}^{+}$escape rate is a factor of 100 below the value derived by Lundin et al. (1989). The charge-exchange reactions seem to be of minor importance for the oxygen loss of the martian atmosphere than previously thought. The NPD data, however, do not allow us to decide whether the oxygen exosphere itself is much thinner than modeled (Krasnopolsky and Gladstone, 1996) (in which case all oxygen loss rates would have to be downscaled) or if the extraction of planetary oxygen (Dubinin et al., 2006a; Lundin et al., 2004) is less efficient than assumed.

6. The actual loss rate of hydrogen of $10^{26} \mathrm{~s}^{-1}$ (Lammer et al., 2005) must be re-examined as well because it is dominated by thermal escape, which has not been directly measured yet. An overestimation of the hydrogen exobase 
density not only results in too high predicted ENA intensities, it also leads to an overestimation of the thermal escape flux $F_{\text {esc }}$ :

$F_{\text {esc }}=0.5 \frac{n_{\mathrm{H}}\left(r_{\text {exo }}\right) U}{2 \sqrt{\pi}}(\lambda+1) \exp (-\lambda)$, where

$U=\sqrt{\frac{2 k T}{m_{\mathrm{H}}}}$, and $\lambda=\frac{G M m_{\mathrm{H}}}{k T r_{\text {exo }}}$.

For the two widely used pairs of density and temperature of the exospheric hydrogen, namely $T=350 \mathrm{~K}, n_{H}=3 \times 10^{10} \mathrm{~m}^{-3}$ according to the UV limb emission measurements done by Anderson and Hord (1971), and $T=200 \mathrm{~K}$, $n_{\mathrm{H}}=10^{12} \mathrm{~m}^{-3}$ according to the model of Krasnopolsky and Gladstone (1996) for solar minimum conditions, Equation (13) yields in both cases a global loss rate of $10^{26} \mathrm{~s}^{-1}$. The same thermal escape rate is calculated for the parameters $T=1000 \mathrm{~K}, n_{\mathrm{H}}=6 \times 10^{9} \mathrm{~m}^{-3}$ derived by Galli et al. (this issue). The effects of the lower density and of the increased temperature compared to the model of Krasnopolsky and Gladstone (1996) cancel each other. The ionospheric escape rate of hydrogen was predicted to reach $10^{26} \mathrm{~s}^{-1}$ as well (Lichtenegger and Dubinin, 1998) but probably this number has to be downscaled since in 2004 the upper limit of the total H-ENA production rate, as well as the surface density of the neutral hydrogen exosphere, are found to be one order of magnitude below the model estimate of Lichtenegger et al. (2002) or Gunell et al. (2006). Recent IMA measurements from 2005 of planetary pick-up protons (Dubinin et al., 2006c) show $\mathrm{H}^{+}$fluxes between $10^{5}$ and $10^{6} \mathrm{~cm}^{-2} \mathrm{~s}^{-1}$ in the outer magnetosheath, which is consistent with a neutral hydrogen exobase density $n_{\mathrm{H}} \leq 10^{11} \mathrm{~m}^{-3}$, but an estimate of the global escape rate of $\mathrm{H}^{+}$from IMA data is not yet available. In any case the hydrogen loss rate due to the production of ENAs is orders of magnitude lower than the thermal escape rate.

7. It was never suggested that the production of ENAs might be the dominant escape process for oxygen or hydrogen at Mars, but given the ENA measurements done with NPD we can even conclude that it is completely negligible compared to other loss processes. Charge-exchange processes in general are of minor importance for the atmospheric loss of hydrogen. ENA measurements combined with UV limb emission measurements and independent ion measurements show a consistent picture of a thin martian exosphere at solar minimum.

\section{Acknowledgments}

The ASPERA-3 experiment on the European Space Agency (ESA) Mars Express mission is a joint effort between 15 laboratories in 10 countries, all sponsored 
by their national agencies. We thank all these agencies as well as the various departments/institutes hosting these efforts. This work is supported by the Swiss National Science Foundation.

\section{References}

Anderson, D. E., and Hord, C. W.: 1971, JGR 76(28), 6666.

Barabash, S., Kallio, E., Lundin, R., and Koskinen, H.: 1995, JGR 100(A11), 21307.

Barabash, S., Holmström, M., Lukyanov, A., and Kallio, E.: 2002, JGR 107(A10), 1280.

Barabash, S., et al.: 2004, ASPERA-3: analyser of space plasmas and energetic ions for Mars Express, 121-139, in Mars Express: the scientific payload. ESA SP-1240, ed. by Andrew Wilson, Noordwijk, Netherlands.

Barabash, S., and Lundin, R.: 2006, Icarus 182, 301.

Barabash, S., Fedorov, A., Lundin, R., and Sauvaud, J.-A.: 2006, Science, submitted.

Brain, D.: 2006, IMF Draping Direction at Mars, http://sprg.ssl.berkeley.edu/brain/rsrch/drapingdirxn.html.

Carlsson, E., et al.: 2006, Icarus 182, 320.

Chassefière, E., and Leblanc, F.: 2004,P\&SS 52, 1039.

Dubinin, E., et al.: 2006a, Icarus 182, 337.

Dubinin, E., et al.: 2006b, Icarus 182, 343.

Dubinin, E., Fränz, M., Woch, J., Barabash, S., Lundin, R., and Yamauchi, M.: 2006c, Hydrogen exosphere at Mars, pickup protons and their acceleration at the bow shock, GRL, in press.

Futaana, Y., et al.: 2006a, Icarus 182, 424.

Futaana, Y., et al.: 2006b, Icarus 182, 413.

Galli, A., et al.: 2006, ApJ 644, 1317.

Galli, A., Wurz, P., Lammer, H., Lichtenegger, H. I. M., Lundin, R., Barabash, S., et al.: Space Sci. Rev., this issue, doi: 10.1007/s11214-006-9089-7.

Gunell, H., Holmström, M., Barabash, S., Kallio, E., Janhunen, P., Nagy, A.F., et al.: 2006, P\&SS 54, 117.

Holmström, M., Barabash, S., and Kallio, E.: 2002, JGR 107(A10), 1277.

Kallio, E., Luhmann, J. G., and Barabash, S.: 1997, JGR 102(A10), 22183.

Kallio, E., et al.: 2006, Icarus 182, 448.

Kim, J., Nagy, A. F., Fox, J. L., and Cravens, T. E.: 1998, JGR 103(A12), 29339.

Krasnopolsky, V. A., and Gladstone, G. R.: 1996, JGR 101(A7), 15765.

Krasnopolsky, V. A.: 2002, JGR 107(E12), 5128.

Lammer, H., Selsis, F., Penz, T., Amerstorfer, U. V., Lichtenegger, H. I. M., Kolb, C., et al.: 2005, in Tokano, T. (ed.), Advances in Astrobiology and Biogeophysics, Springer-Verlag, Berlin, Germany, p. 25.

Lichtenegger, H. I. M., and Dubinin, E.: 1998, EP\&S 50, 445.

Lichtenegger, H. I. M., Lammer, H., and Stumptner, W.: 2002, JGR 107(A10), 1279.

Lichtenegger, H. I. M., Lammer, H., Kulikov, Yu. N., Kazeminejad, S., Molina-Cuberos, G. H., Rodrigo, R., et al:: Space Sci.Rev., this issue, doi: 10.1007/s11214-006-9082-1.

Lundin, R., Zakharov, A., Pellinen, R., Borg, H., Hultqvist, B., Pissarenko, N., et al.: 1989, Nature 341, 609-612.

Lundin, R., et al.: 2004, Science 305, 1933.

Macias, A., Riera, A., and Yañez, M.: 1983, Phys. Rev. A 27(1), 213.

Nier, A. O., and McElroy, M. B.: 1977, JGR 82(28), 4341. 
Wurz, P.: 2000, in Scherer, K., et al. (ed.), Detection of Energetic Neutral Particles, in The Outer Heliosphere: Beyond the Planets, Katlenburg-Lindau: Copernicus-Gesellschaft e.V., 251.

Yamauchi, M., Futaana, Y., Fedorov, A., Dubinin, E., Lundin, R., Sauvaud, J.-A., et al.: Space Sci. Rev., this issue, doi: 10.1007/s11214-006-9090-1. 